\title{
Does Affective Polarization Undermine Democratic Norms or Accountability? Maybe Not*
}

\author{
David E. Broockman ${ }^{\dagger} \quad$ Joshua L. Kalla ${ }^{\ddagger} \quad$ Sean J. Westwood ${ }^{\S}$ \\ First draft: December 21, 2020 \\ This draft: January 13, 2022
}

Short title: Does affective polarization undermine democratic norms or accountability?

\section{Forthcoming, American Journal of Political Science}

\begin{abstract}
Scholars warn that affective polarization undermines democratic norms and accountability: they speculate that if citizens were less affectively polarized, they would be less likely to endorse norm violations, overlook copartisan politicians' shortcomings, oppose compromise, adopt their party's views, or misperceive economic conditions. We advance reasons to doubt that affective polarization influences political choices. We support this argument with five experiments which manipulate citizens' affective polarization with multiple approaches. We then trace the downstream consequences of manipulating citizens' affective polarization, such as their reactions to information about their actual representatives in Congress. In our experiments (total $N=12,341$ ), we 'rewind' the equivalent of three decades of change in affective polarization, but find no evidence that these changes influence many political outcomes - only general questions about interpersonal attitudes. Our results suggest caution about assuming that reducing affective polarization would meaningfully bolster democratic norms or accountability.
\end{abstract}

Keywords: affective polarization, accountability, democratic norms, survey experiments

The data and materials required to verify the computational reproducibility of the results, procedures and analyses in this article are available on the American Journal of Political Science Dataverse within the Harvard Dataverse Network, at: https: / / dataverse.harvard.edu/dataset. xhtml?persistentId=doi: $10.7910 / \mathrm{DVN} / 3 \mathrm{AOBP} 2$.

*This research was approved by the IRBs at the University of California, Berkeley, Stanford University, Yale University, and Dartmouth College. Broockman acknowledges the Civic Health Project for support.

$\dagger$ Associate Professor, Travers Department of Political Science, University of California, Berkeley. 210 Social Sciences Building 1950, Berkeley, CA 94720. dbroockman@berkeley . edu.

¥Assistant Professor, Department of Political Science and Department of Statistics and Data Science, Yale University. 115 Prospect Street, New Haven, CT 06520. josh.kalla@yale.edu

${ }^{\S}$ Associate Professor, Department of Government, Polarization Research Lab, Dartmouth College, 3 Tuck Mall, Hanover, NH 03755. sean.j.westwoodedartmouth.edu. 
Affective polarization-citizens' more negative sentiment towards opposing political parties than their own (Iyengar, Sood and Lelkes 2012) — has been growing worldwide. Research on this trend constitutes one of the most influential literatures in contemporary social science and has sown alarm across disciplines (e.g., Finkel et al.2020).

The gravest worries this literature raises are for democracy: that affective polarization has political consequences, such as changing the actions citizens incentivize politicians to take, or shifting the norms to which they expect their representatives to adhere. In Table 1, we give examples of over a dozen studies that express concern about such potential political consequences of affective polarization.

Surprisingly, however, research investigating potential political consequences of affective polarization is scarce. Existing literature has generally simply asserted that affective polarization has downstream consequences for political decisions, with scant attention paid to the theoretical process by which this might happen and with little supporting empirical evidence (but see Kingzette et al. 2021). In other words, speculation is rife about the political implications of affective polarization, but we actually "know little" (Druckman et al. 2020, p. 9).

In this article, we remedy this dearth of theory and evidence.

Theoretically, returning to the insights of foundational research on affect and decision-making, we describe reasons to be skeptical that affective polarization's consequences would spill over into political judgments. Empirically, we then address one of the main challenges faced by previous research. Previous research has had difficulty investigating the political consequences of affective polarization because of the potential for omitted variable bias: because affect serves to summarize other considerations, people with different levels of affective polarization likely differ in other ways, confounding comparisons between them. To estimate the causal effects of affective polarization on political attitudes, we conduct a series of experiments which manipulate Americans' levels of affective polarization and then probe potential downstream consequences, such as consequences for how they react to information about their 
Table 1: Example Speculation On Political Implications of Affective Polarization in Prior Work

\begin{tabular}{|c|c|}
\hline & Quote \\
\hline $\begin{array}{l}\text { Electoral } \\
\text { Account- } \\
\text { ability }\end{array}$ & 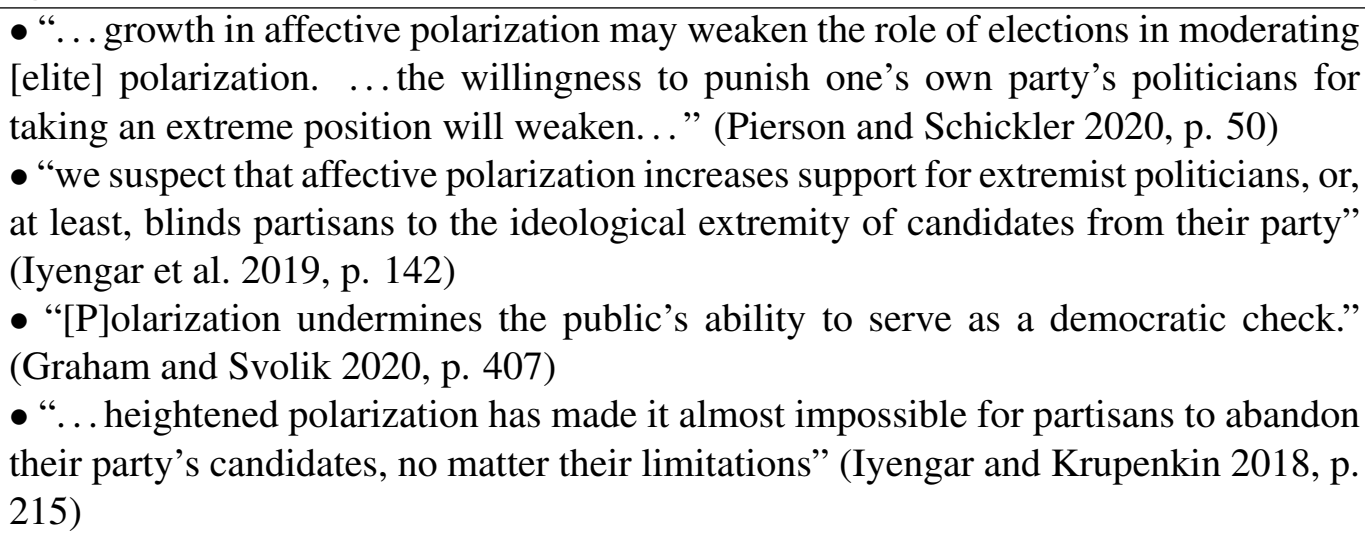 \\
\hline $\begin{array}{l}\text { Adopting } \\
\text { Party's } \\
\text { Policy } \\
\text { Attitudes }\end{array}$ & $\begin{array}{l}\text { - "For the mass public, we suspect that affective polarization increases partisans" } \\
\text { willingness to conform to their party's policy positions" (Iyengar et al. 2019, p. 142) }\end{array}$ \\
\hline $\begin{array}{l}\text { Legislative } \\
\text { Bipartisan } \\
\text {-ship }\end{array}$ & $\begin{array}{l}\text { - "holding opposing partisans in contempt ... precludes innovative cross-party } \\
\text { solutions and mutually beneficial compromises" (Finkel et al. } 2020, \text { p.533) } \\
\text { • "... a affective polarization makes governance more difficult." (Levendusky 2018, p. } \\
\text { 59) }\end{array}$ \\
\hline $\begin{array}{l}\text { Democratic } \\
\text { Norms }\end{array}$ & $\begin{array}{l}\text { - "The cumulative effect of severe polarization ... is a deterioration in the quality } \\
\text { of democracy, leading to backsliding, illiberalism, and in some cases reversion to } \\
\text { autocracy." (McCoy and Somer 2019, p. 258) } \\
\text { - "As affective polarization increases, partisans may become more likely to ignore } \\
\text { democratic norms..." (Kingzette et al. } 2021, \text { p. 2) } \\
\text { - "affective polarization can have grave ramifications... Partisanship appears to now } \\
\text { compromise the norms and standards we apply to our elected representatives, and } \\
\text { even leads partisans to call into question the legitimacy of election results" (Iyengar } \\
\text { et al. 2019. p. 143) }\end{array}$ \\
\hline $\begin{array}{l}\text { Condition } \\
\text { Perceptions }\end{array}$ & $\begin{array}{l}\text { - "These findings underscore the challenges that affective polarization poses for } \\
\text { governance. In a polarized America, citizens may be willing to tolerate poor } \\
\text { economic performance from their own party, or fail to reward the other side for } \\
\text { apparently good economic stewardship." (Freeder } 2020, \text { p. } 28) \\
\text { - "[T]he increased level of affective polarization poses considerable challenges to } \\
\text { the democratic process. Partisan bias in perceptions of economic conditions means } \\
\text { that voters will fail to credit opposing-party incumbents when the economy grows } \\
\text { under their stewardship and fail to penalize in-party incumbents whose economic } \\
\text { performance is suspect." (Iyengar, Sood and Lelkes } 2012, \text { p. } 428)\end{array}$ \\
\hline
\end{tabular}


representatives. In our first four experiments, we use a trust game manipulation which produces very large effects on affective polarization as measured in the same manner as the literature; the exogenous differences in affective polarization we create are similar in size to three decades of increases in affective polarization in the United States. We also conduct a fifth experiment as a robustness check to examine the downstream effects of three alternative and conceptually distinct approaches for manipulating affective polarization.

Deploying these paradigms across five surveys and $N=12,341$ respondents, we investigate the causal effects of affective polarization on a variety of downstream outcomes, employing a broad suite of measurement strategies. First, supporting previous literature's findings, we show that increasing affective polarization has large downstream effects on general interpersonal questions-general questions about other people that do not involve political candidates, institutions, or issues, e.g., stating general discomfort with having outpartisan friends. We then investigate effects in five political domains identified in prior literature (see Table 1): electoral accountability (measured by levels of party loyalty and how individuals react to information about their actual representatives), adopting one's party's policy positions, support for legislative bipartisanship, support for democratic norms, and perceptions of objective conditions. Our results run contrary to the literature's widespread speculation: in these political domains, our estimates of the causal effects of reducing affective polarization are consistently null across outcomes and approaches for manipulating affective polarization.

In concluding, we discuss potential alternative explanations, remaining limitations, and potential extensions. Most importantly, our results suggest lessons for those seeking to bolster support for democratic norms or bolster democratic accountability: our results suggest that efforts to reduce affective polarization may not be the most effective strategy for achieving these goals. 


\section{Theoretical Framework}

In this article, we define affective polarization as traditionally conceptualized, the difference in affect one has for one's own party minus the outparty (Iyengar, Sood and Lelkes 2012). 1 Theories of affect conceptualize it as representing an "overall summary evaluation" (Ottati and Wyer 1993, p. 302), collapsing judgments about many dimensions of an attitude object to a single dimension of overall liking or disliking. Affect is therefore often used as a heuristic to simplify a complex judgment into a simpler question: do I like this attitude object (Slovic et al.|2002)?

The literature that speculates affective polarization influences political outcomes has not laid out a theoretical argument for why this might occur. However, building on traditional theories of affect, such speculation could be consistent with a model like that shown in Figure 1a. Under this model, a number of considerations influence voters' affect towards the parties. Then, when making political choices, such as whether to re-elect their member of Congress, voters might simply rely on their affect towards the parties as a heuristic.

We explicate two reasons to be skeptical this would usually occur.

First, when forming judgments, individuals seek to use heuristics that are both easily accessible and as relevant to the judgment as possible. As Ottati and Wyer (1993, p. 298) note, "evaluations of an object might not be based on affective reactions at all" when individuals have more relevant dimensions to rely on. When forming judgments about objects related to but distinct from parties, such as candidates or norms, there may be other easy-to-access attributes (and competing heuristics) that are usually more relevant than one's affect towards a party. One could therefore imagine partisan affect manifesting in abstract survey questions about whether one would be unhappy having an outpartisan neighbor (as there are no other dimensions of judgment available) but not evaluations of an actual outpartisan neighbor or an actual outpartisan

\footnotetext{
${ }^{1}$ Some research has considered affective polarization in the context of broader constructs, such as partisan identity. When we refer to affective polarization, we refer only to the original definition and not these broader constructs. Additionally, our manipulation checks distinguish between affect towards citizens and elites, and we find increases in affective polarization towards both (Figure 2 ).
} 
Figure 1: Observational Equivalence Between Literature's Speculation and Alternative Hypotheses

(a) Literature's Speculation: Affective Polarization Affects Political Choices

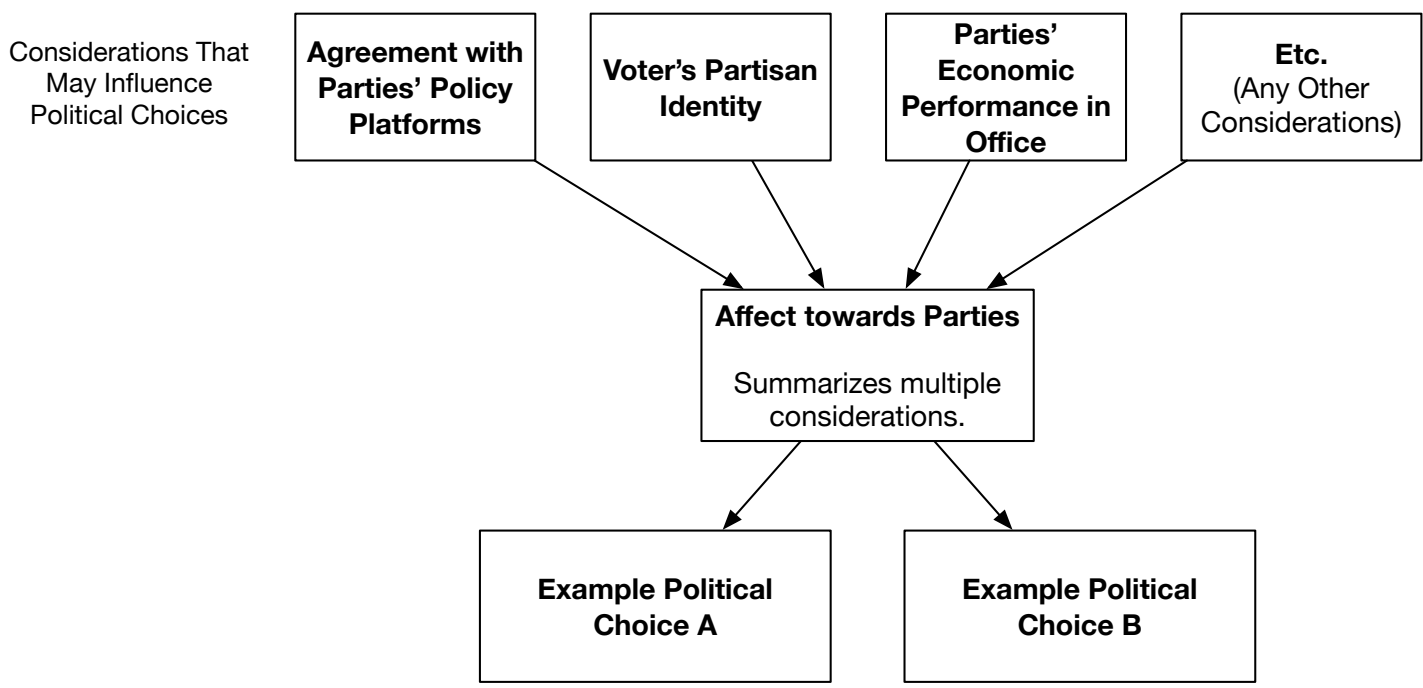

(b) Alternative: Other Considerations Influence Both Affect, Other Political Choices (Example Choice A)

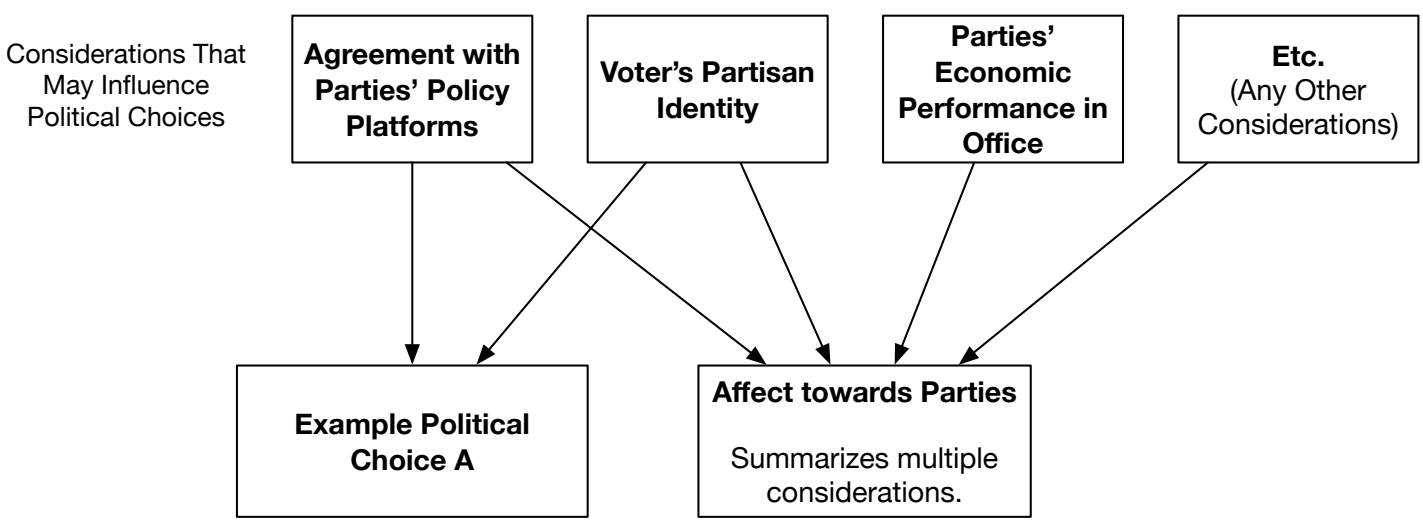

politician-cases when other information is available. ${ }^{2}$

A second reason to doubt that individuals make different choices due to affective polarization is that individuals must give up other things they value in order to do so- that is, it is costly for voters to allow affective polarization to influence their judgments. This reflects a famous insight in economics (Becker 1957): if tastes for one dimension cause us to change our choice from option A to option B, we must pay the cost of giving up whatever else it is we originally liked about

\footnotetext{
${ }^{2}$ This does not imply that there would be no partisan bias in such encounters, it could simply arise from another source, such as partisan identity.
} 
option A instead of B. Put differently, presuming people have reasons to make the choices they do, in order for them to make a different choice (i.e., allowing affective polarization to affect their judgments), they must decide to surrender whatever made their original choice attractive. The existence of such a trade-off is a necessary condition for affective polarization to cause people to make different choices than they would otherwise make. However, there is room to doubt that voters would make such trades.

This second argument would also predict that affective polarization may have some effect on answers to abstract questions about whether one would be happy to interact with outpartisans, as there exist limited trade-offs when answering such an abstract question. However, affective polarization may have less of an effect on interactions with specific outpartisans for whom respondents have more information because trade-offs would exist.

Of course, it remains an empirical question as to whether or not citizens in fact do rely on partisan affect when forming judgments in which partisanship is only one possible criteria, and whether they would be willing to make the trade-offs necessary to do so. However, despite many scholars asserting that affective polarization causes a variety of political outcomes (see Table 1), there is surprisingly little empirical research exploring the role of affective polarization in explicitly political contexts. Many reviews of the literature explicitly note this evidentiary lacuna (e.g., Iyengar et al. 2019; Druckman et al.|2020).

Our theoretical framework indicates why such evidence has been difficult to muster: observational relationships between affective polarization and other outcomes, both cross-sectional and over-time, do not imply affective polarization has a causal affect on these outcomes. As shown in Figure $1 \mathrm{~b}$, insofar as affect serves to summarize other considerations, these other considerations may influence both affective polarization and political choices, leading the two to correlate even if affective polarization has no effect on political choices-a classic case of omitted variable bias.

Nevertheless, understanding whether affective polarization impacts political judgments has 
important implications for efforts to reverse negative trends in democracies: would decreasing affective polarization bolster accountability and support for democratic norms, or does affective polarization correlate with these outcomes merely because it correlates with other more consequential dimensions? To answer this question, researchers must locate or produce an exogenous source of variation in affective polarization and then trace its impact on these downstream outcomes of interest. We do just that.

\section{Data and Research Design: Surveys 1-4}

We trace the downstream impacts of affective polarization on a broad variety of political outcomes across five surveys with a total sample size of $N=12,341$. Our surveys deploy a two-stage process in which we first randomly assign a task which changes respondents' level of affective polarization and then present a number of survey items potentially downstream of affective polarization. This design facilitates a causal analysis of the downstream consequences effect of affective polarization.

\section{Data in Surveys 1-4}

Our primary data is drawn from four surveys conducted in 2019 and 2020 using the online survey vendor Dynata. In order to be eligible, participants needed to provide their informed consent, identify as a Democrat or a Republican (including leaners), pass a pre-treatment attention check, and demonstrate understanding of the trust game (described below). All these criteria were assessed prior to random assignment. These surveys resulted in a total of $N=9,837$ completed responses. They were conducted in October 2019 (Survey $1 N=1,684$ ), December 2019 (Survey $2 N=2,499$ ), December 2019 (Survey $3 N=3,519$ ), and December 2020 (Survey $4 N=2,135$ ). The studies were all pre-registered ${ }^{3}$

Appendix Table A3 presents the demographics of the survey samples relative to the 2019 CCES, finding broad similarity. The largest difference is that our sample is slightly more

\footnotetext{
${ }^{3}$ See Online Appendix B.
} 
educated, potentially because we require our respondents to successfully complete an attention check and understand the trust game. Survey questions appear in Appendix D. Although all surveys contained the trust game before the outcome measures, different surveys contained different outcome measures. Appendix Table A2 lists which items appeared on which surveys and the order in which they appeared. Appendix Section D.3 discusses survey attrition.

\section{Inducing Variation in Affective Polarization with a Trust Game in Surveys}

\section{1-4}

After collecting demographics, surveys 1-4 prompted participants to engage in a task from Westwood and Peterson (2020) which manipulates affective polarization.

This task uses a scripted set of allocations in a modified trust game. In typical trust games, there are two players. Player 1 receives a cash allocation and is instructed to give "some, all, or none" of the money to Player 2. The player is also told that the researchers will triple any amount Player 1 gives to Player 2. Furthermore, Player 2 can return some, all, or none of the money back to Player 1. Therefore, the more Player 1 expects reciprocity from Player 2, the more money they should allocate to Player 2 in anticipation they will receive a larger sum in return.

Studies 1-4 deploy a modified version of this game. First, we always make participants take the role of Player 2. This means they always first observe an allocation another player makes to them. Second, across three consecutive rounds of game play, participants are told they are interacting with three other respondents of the opposite political party who have each been allocated $\$ 10.4$ However, they are in fact interacting with computerized opponents who offer pre-determined allocations. Participants randomized to the Positive Experience condition receive allocations from Player 1 of $\$ 8, \$ 7$ and $\$ 8$ (tripled to $\$ 24, \$ 21$ and $\$ 24$ ) respectively across the three rounds of the game. However, those in the Negative Experience condition receive $\$ 0$ allocations from Player 1 in all three rounds. Following each round, in both conditions, participants are told the other player said that they made their allocation due to the player's own

\footnotetext{
${ }^{4}$ The opponent's other profile attributes (gender, age, and income) were randomly assigned in each round of play.
} 
partisanship. At the end of the game, participants see a summary of the results for all rounds. Consistent with our ex ante instructions to respondents, after they completed the survey we paid respondents a bonus equal to 0.05 times the amount they won.

In the survey, participants read instructions, saw three example rounds to ensure they understood the game, and completed two comprehension questions $\sqrt[5]{5}$ They then participated in the trust game. All game instructions are given in Online Appendix D.4.

\section{Manipulation Checks}

This manipulation produced large changes in affective polarization in all four of our surveys, as assessed by the main measures of affective polarization employed in the literature. In particular, participants assigned to the Positive Experience condition rated outpartisan citizens and outpartisan politicians and elected officials much more positively than those in the Negative Experience condition.$^{6}$ (Evidence presented in Appendix $\mathrm{E}$ indicates that the difference between the conditions is driven by decreases in affective polarization in the Positive Experience condition.) For example, in Survey 1, the average feeling thermometer difference for in- minus out-party citizens in the Negative Experience group was 36.3, but in the Positive Experience group it shrunk to 23.8. Across our four surveys, the average difference in affective polarization towards other citizens across the Positive and Negative Experience conditions is 14.3 degrees, with most of this coming from changes in affect towards outpartisans (15.5 degrees). The effect on affective polarization towards political elites (measured only in Survey 4) is 9.8 degrees. These differences are approximately equivalent to 'rewinding' over three decades of increasing affective polarization in the United States.7

The top row of Figure 2 visualizes these effects in standard deviations. The coefficients

\footnotetext{
${ }^{5}$ If respondents missed a comprehension question, participants were given the answer and asked the questions again. Those failing the questions three times were removed from the survey prior to random assignment.

${ }^{6}$ These feeling thermometer items ask about "People who are Democrats/Republicans" and "Democratic/Republican Politicians and Elected Officials" in general, and do not reference the individual players in the game.

${ }^{7}$ The total increase in affective polarization Iyengar, Sood and Lelkes (2012, Table A1) observe from 1978 to 2008 is 15.35 degrees for Democrats and 10.16 degrees for Republicans, for an average of 12.76.
} 
estimate the effect of the Positive Game Experience condition relative to the Negative Game Experience condition. Mirroring Iyengar, Sood and Lelkes's (2012) findings regarding changes over time in the electorate, we see small changes in sentiments towards inpartisans, but large increases in sentiments towards outpartisans as a result of the Positive Game Experience condition. Importantly, the intervention also meaningfully affected affective polarization towards "politicians and candidates." Finally, unsurprisingly, we show that respondents were much more likely to say they were treated fairly in the trust game if in the Positive Experience condition.

Figure 2: Effect of Positive Trust Game Experience on Manipulation Checks
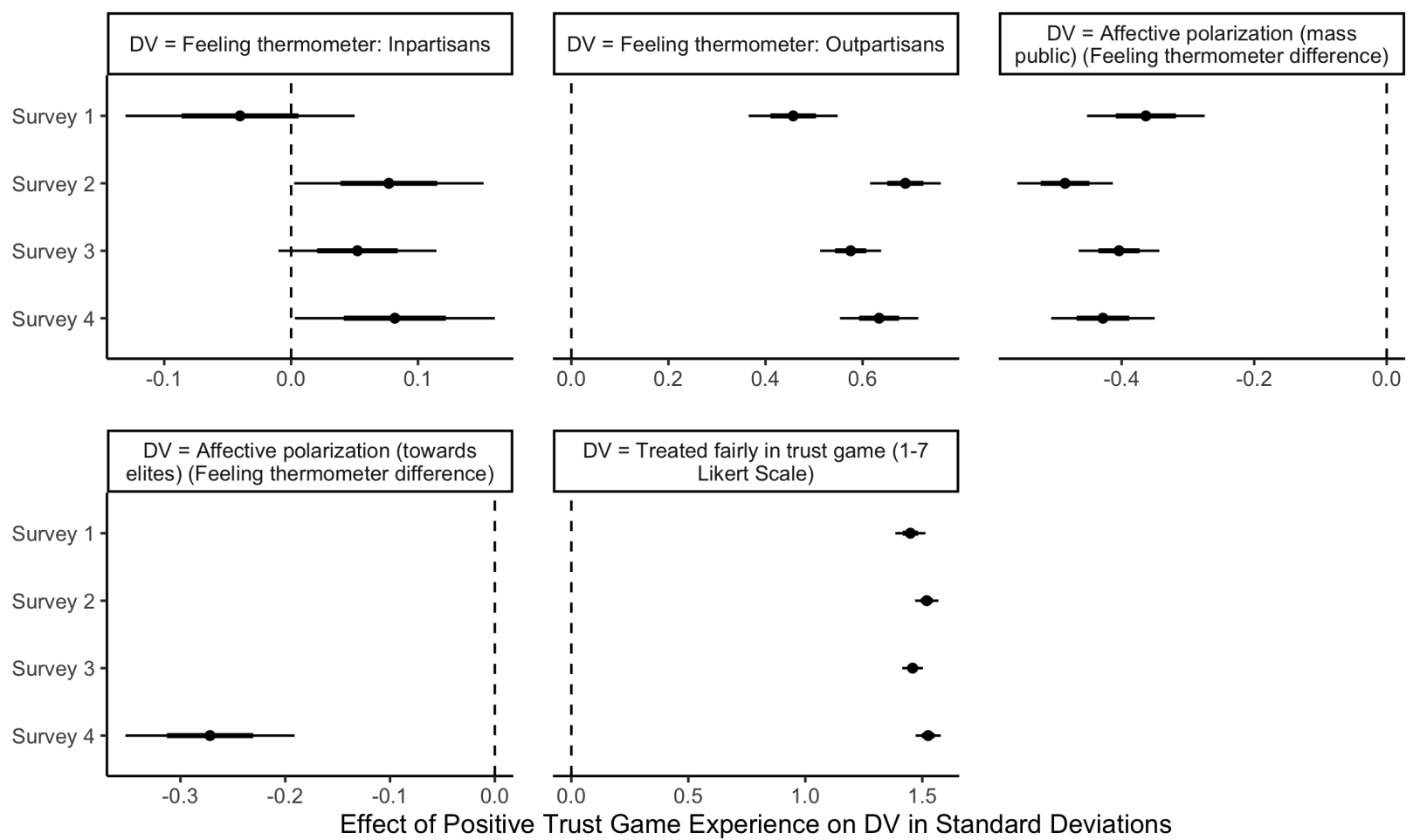

Notes: Point estimates are surrounded by one standard error (thick tails) and $95 \%$ confidence intervals (thin tails). Point estimates are from multivariate regressions controlling for preregistered covariates. 


\section{Survey 1-4 Results: Downstream Consequences of Affective}

\section{Polarization}

We next exploit these experimentally-induced reductions in affective polarization to analyze its downstream consequences across a variety of outcomes.

\section{Apolitical, Interpersonal Items: General Social Distance Measures}

We first examine the causal effects of affective polarization in an apolitical domain where prior research has also documented its effects: general social distance.

Figure 3 reports the effects of the treatment on a set of general social distance measures, most from Iyengar, Sood and Lelkes (2012) and Druckman and Levendusky (2019). These items probe generalized discomfort with social interactions or ties with outpartisans. The items do not refer to the particular partisans in the trust game. We asked these items on Survey 4.

As with the all the remaining Figures in the article, we orient the dependent variables such that negative coefficients would correspond with the literature's prevailing predictions about what the impact would be of reducing affective polarization. Therefore, in this case, the negative estimates capture decreased discomfort with social interactions with outparty members.

As Figure 3 shows, we observe large and statistically significant decreases on all the social distance items as a result of the positive trust game condition. The first coefficient shows an index we pre-registered of all four of these items; the effect on this index is also significant. The treatment strongly affected all of the items we drew from previous literature.

\section{Electoral Accountability}

We next turn to evaluating affective polarization's downstream consequences in political domains. Perhaps the concern scholars most commonly articulate about the political consequences of affective polarization is that it encourages voters to loyally vote for their party's candidates, no matter their shortcomings. Many scholars express some version of this concern 
Figure 3: Effect of Reducing Affective Polarization on Social Distance Items (Survey 4)

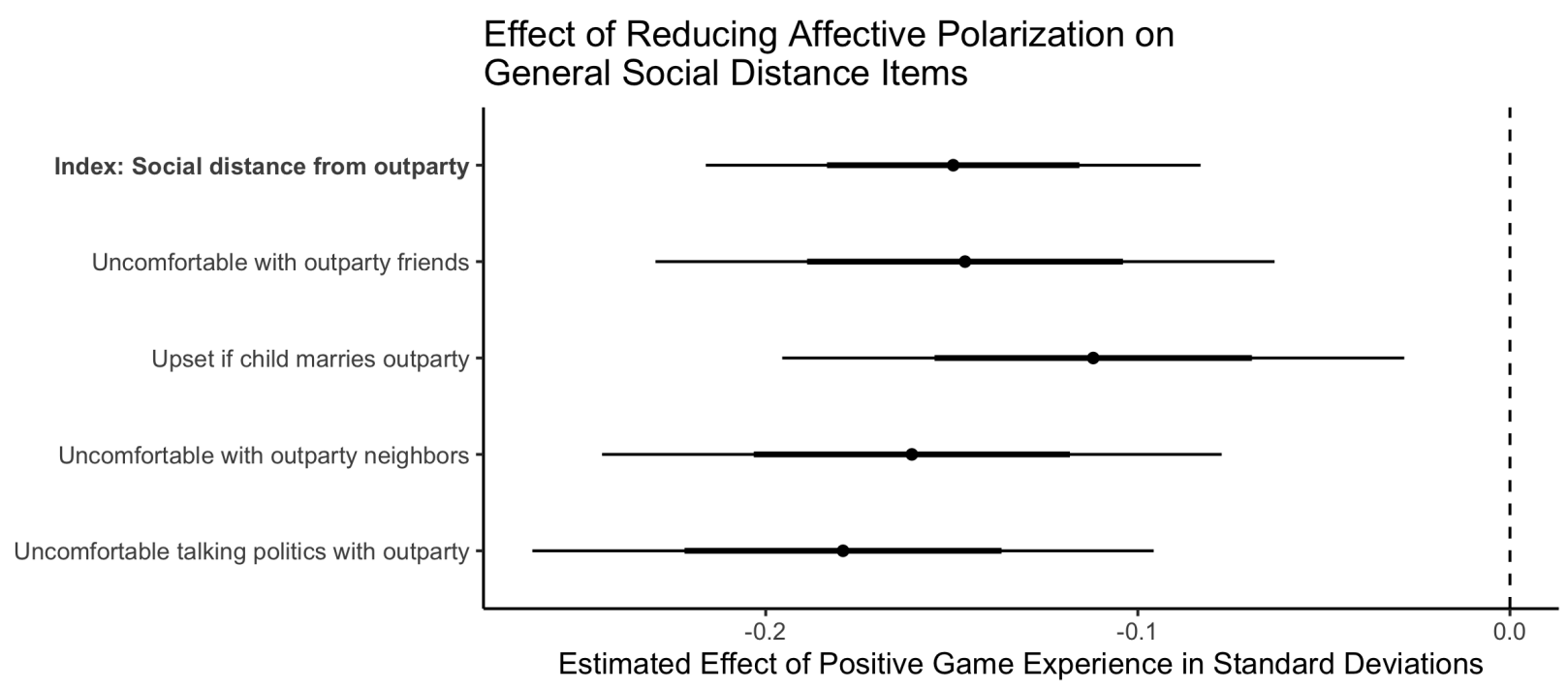

Notes: Point estimates are surrounded by one standard error (thick tails) and $95 \%$ confidence intervals (thin tails). Point estimates are from multivariate regressions controlling for preregistered covariates.

(see Table 11), such as concern that affective polarization "has made it almost impossible for partisans to abandon their party's candidates, no matter their limitations" (Iyengar and Krupenkin 2018, p. 215).

Following from Little, Schnakenberg and Turner's (2022) framework, we consider two possible ways this concern might manifest: divergence, in which affective polarization simply increases party loyalty; and desensitization, in which affective polarization weakens voters' responses to information about incumbent's actions.

We sought to assess these consequences of affective polarization with an experiment with a high degree of realism. In particular, in Surveys 1 and 3, we first asked respondents for their zip code so that we could determine their Congressional district and, in turn, their Member of Congress (MC). We next asked them whether they personally supported or opposed a series of bills that Congress had previously voted on (see Appendix $\mathrm{D}$ for full text). Respondents then played the trust game and were randomized to have a positive or negative experience. 
Finally, we showed respondents how their MC actually voted on several bills, randomizing whether we showed respondents congruent votes-up to 3 votes where they agreed with how their MC voted-or incongruent votes — up to 3 votes where they disagreed with how their MC voted. We determined the congruent and incongruent votes by comparing respondent's pre-treatment stated preferences on each issue to how their MC actually voted. That is, respondents were randomized to learn about real votes their representative in Congress had actually cast that were either in line with respondents' pre-treatment issue positions or out of step with respondents' pre-treatment positions. In Survey 3, we also included a pure control group where respondents received no information about how their MC voted.

We concluded by asking a series of questions capturing vote intention in the next Congressional election and approval of their MC. As we pre-registered, we combine these into a MC Approval Index .8 our primary outcome. This index is standardized to have mean zero and standard deviation one in each survey.

Respondents were told their MC's party when they learned about their votes and the MC's party affiliation was again given in the survey question asking for their vote intentions and approval.

This design produced the $2 x 3$ factorial design shown in Table 2, The first factor, shown at the top, is the affective polarization manipulation: whether respondents had a Positive or Negative Game Experience, which manipulated their levels of affective polarization. The second factor, shown at left, is the MC vote manipulation: whether respondents were shown votes their MC cast which were either congruent or incongruent with their own views, or no votes. Respondents are therefore assigned to one of the six cells shown in the center of Table 2

\section{Divergence: Does Affective Polarization Increase Party Loyalty?}

Our first test is for divergence: if voters were less affectively polarized, would they be less loyal to their own party, and more willing to vote for the outparty? To test this, for the moment we set

\footnotetext{
${ }^{8}$ As we pre-registered, we standardize these three questions and compute an additive index of them. In Survey 1 , $\alpha=0.93$; in Survey 3, $\alpha=0.83$.
} 
Table 2: 2x3 Design: MC Accountability Experiment

\begin{tabular}{|c|c|c|c|c|}
\hline & $\begin{array}{c}\text { Affective Polariza } \\
\text { Column 1: } \\
\text { Negative Game } \\
\text { Experience (Higher } \\
\text { Affective Polarization) }\end{array}$ & $\begin{array}{c}\text { ation Manipulation } \\
\text { Column 2: } \\
\text { Positive Game } \\
\text { Experience (Lower } \\
\text { Affective Polarization) }\end{array}$ & $\begin{array}{c}\text { Difference } \\
\text { Between } \\
\text { Columns } 1 \text { and } 2\end{array}$ \\
\hline $\begin{array}{c}\text { Member } \\
\text { of } \\
\text { Congress } \\
\text { Vote } \\
\text { Manip- } \\
\text { ulation }\end{array}$ & $\begin{array}{c}\text { Row 1: } \\
\text { Show Congruent } \\
\text { Votes (Votes } \\
\text { Respondent } \\
\text { Agrees With) } \\
\text { Row 2: } \\
\text { Show Incongruent } \\
\text { Votes (Votes } \\
\text { Respondent } \\
\text { Disagrees With) } \\
\text { Row 3 } \\
\text { (Survey } 3 \text { Only): } \\
\text { Show No Votes } \\
\text { (Control) }\end{array}$ & Cell 3 & 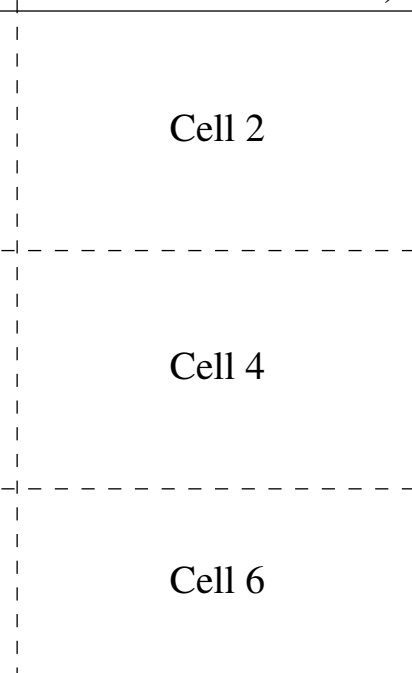 & $\begin{array}{c}\text { Effect of Reducing } \\
\text { Affective Polarization, } \\
\text { Given Respondent } \\
\text { Shown Congruent } \\
\text { Votes } \\
\text { Effect of Réducing } \\
\text { Affective Polarization, } \\
\text { Given Respondent } \\
\text { Shown Incongruent } \\
\text { Votes } \\
-\overline{E f f e c t} \text { of Re } \overline{\text { Reducing }}-\overline{-} \\
\text { Affective Polarization, } \\
\text { Given Respondent } \\
\text { Shown No Votes }\end{array}$ \\
\hline & $\begin{array}{c}\text { Difference } \\
\text { Between } \\
\text { Rows } 1 \text { and } 3\end{array}$ & $\begin{array}{c}\text { Effect of Showing } \\
\text { Incongruent vs. } \\
\text { Congruent Votes, } \\
\text { Under Higher } \\
\text { Affective Polarization } \\
\text { Effect of Showing } \\
\text { Congruent vs. } \\
\text { No Votes, } \\
\text { Under Higher } \\
\text { Affective Polarization } \\
\text { Effect of Showing } \\
\text { No vs. } \\
\text { Incongruent Votes, } \\
\text { Under Higher } \\
\text { Affective Polarization }\end{array}$ & $\begin{array}{c}\text { Effect of Showing } \\
\text { Incongruent vs. } \\
\text { Congruent Votes, } \\
\text { Under Lower } \\
\text { Affective Polarization } \\
\text { Effect of Showing } \\
\text { Congruent vs. } \\
\text { No Votes, } \\
\text { Under Lower } \\
\text { Affective Polarization } \\
\text { Effect of S Showing } \\
\text { No vs. } \\
\text { Incongruent Votes, } \\
\text { Under Lower } \\
\text { Affective Polarization }\end{array}$ & \\
\hline
\end{tabular}

aside and hold constant the $\mathrm{MC}$ vote manipulation and focus on the main effects of the affective polarization manipulation. In particular, we compare the MC Approval Index in the Positive and Negative Game Experience conditions; i.e., in Table 2, we compare Cells 2, 4, and 6 to Cells 1, 3, and 5 .

Figure 4 shows the results. As in all our Figures, we code the dependent variables such that 
prevailing predictions in the literature about the impact of reducing affective polarization correspond with negative coefficients. In each panel, we show the results for both Survey 1 and Survey 3 separately, followed by the pooled results of both coefficients.

Figure 4: Testing for Divergence: Does Reducing Affective Polarization Reduce Approval of Copartisan MCs or Increase Approval of Outpartisan MCs?

\section{Direct Effect of Reducing Affective Polarization on Approval of Own MC, by MC Party} Coefficients $=$ Table $2($ Cells 2,4 , and 6$)-($ Cells 1,3 , and 5$)$

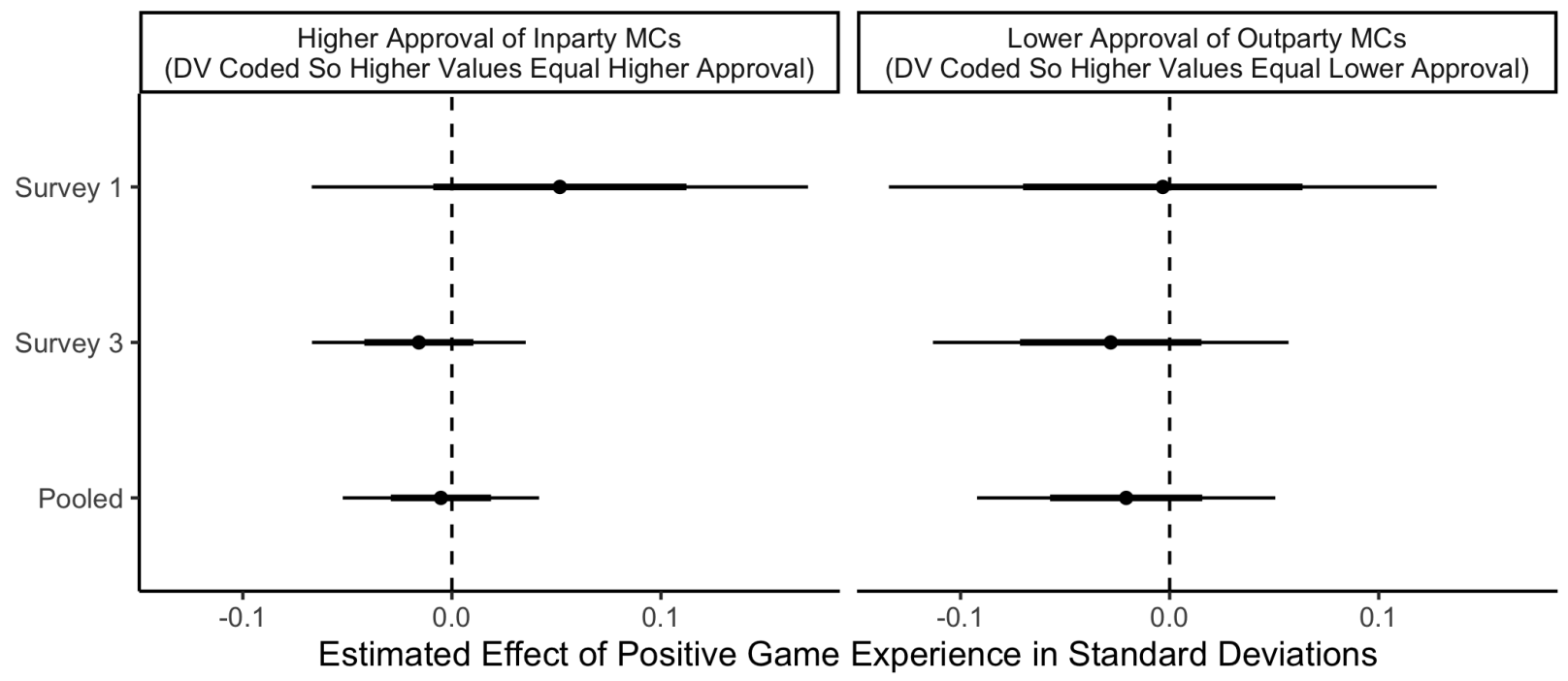

Notes: Point estimates are surrounded by one standard error (thick tails) and 95\% confidence intervals (thin tails). Point estimates are from multivariate regressions controlling for preregistered covariates.

The left panel subsets to respondents with copartisan MCs and tests whether respondents approve less of their copartisan MCs when their level of affective polarization is decreased (e.g., due to decreased party loyalty). If this were the case, we would expect to see negative coefficients, i.e., that reducing affective polarization decreased loyalty to inparty MCs. We do not. The right panel subsets to respondents with outpartisan MCs and shows that decreasing participants' level of affective polarization does not make them disapprove of their outpartisan MCs any less; i.e., we do not see respondents showing decreased hostility to outparty MCs when they are less affectively polarized. The point estimates in both studies in both panels are 
essentially zero, with tight confidence intervals. Reducing affective polarization does not appear to reduce party loyalty in voting.

\section{Desensitization: Does Affective Polarization Weaken Reactions to Information about Politicians' Actions?}

Another, more subtle possibility is that affective polarization weakens voters' reactions to politicians' actions in office. Following Little, Schnakenberg and Turner (2022), we call this desensitization.

To test for this, we first separately estimate the effects of showing respondents incongruent votes their MC cast (i.e., the "Differences Between" rows at the bottom of Table 2) broken down by affective polarization condition. The desensitization hypothesis would predict that the effects of showing respondents information about how their MC voted would be larger once respondents' level of affective polarization is reduced; i.e., in the Positive Game Experience condition.

Consider first the results in Figure 5a, which shows results from Survey 1. The left panel of Figure $5 \mathrm{a}$ plots the effects of showing incongruent (instead of congruent) votes on the MC Approval Index, separated by affective polarization condition. The first coefficient, above "Effect in High Affpol Condition" shows that, given a respondent has been randomly assigned to the High Affective Polarization condition, showing them incongruent instead of congruent votes their MC cast causes them to approve of their MC much less (Cohen's $d=-0.70) .9$

The key test for desensitization is how the effect of showing incongruent instead of congruent votes differs in the experimental condition with lower affective polarization. If the literature's speculation is correct, we should see respondents be more sensitive to information about how their MC voted when they are less affectively polarized. However, the second coefficient shown in Figure $5 \mathrm{a}$ if anything indicates the opposite, as the effect of showing incongruent votes is actually slightly smaller $(d=-0.57)$ when respondents' level of affective polarization is

\footnotetext{
${ }^{9}$ This coefficient compares Cells 3 and 1 in Table 2 This result also indicates our null results are not due to pre-treatment or ceiling/floor effects; respondents' views about their MCs can be changed.
} 
Figure 5: Testing for Desensitization: Does Increasing Affective Polarization Reduce Voters' Sensitivity to Information about their Member of Congress' Votes?

(a) Survey 1: Effect of Showing Incongruent (vs. Congruent) Votes

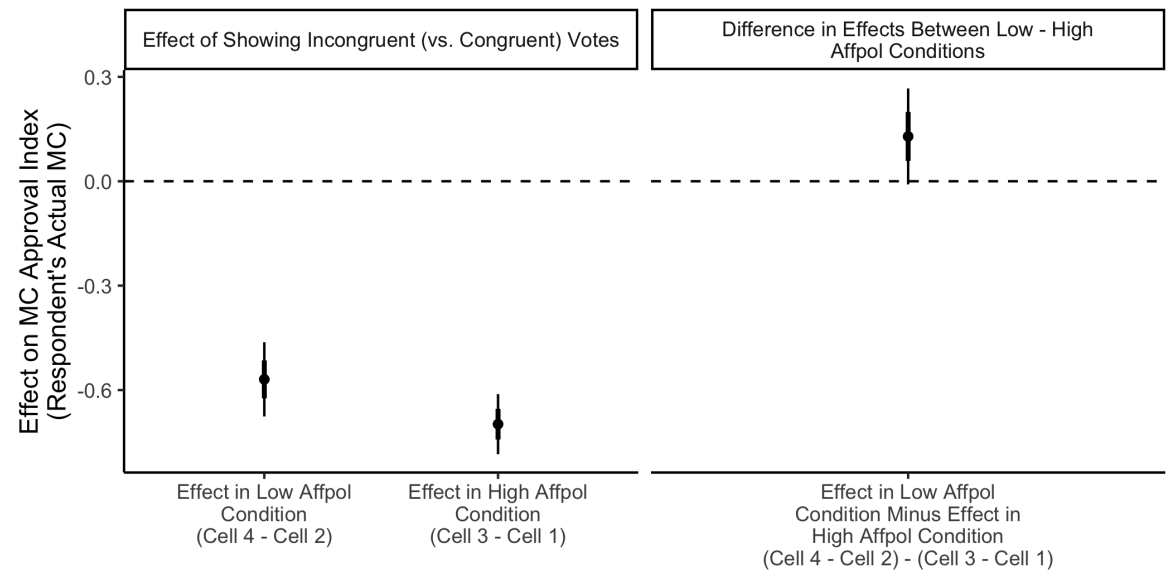

(b) Survey 3: Effect of Showing Incongruent (vs. Not Showing Votes) Votes

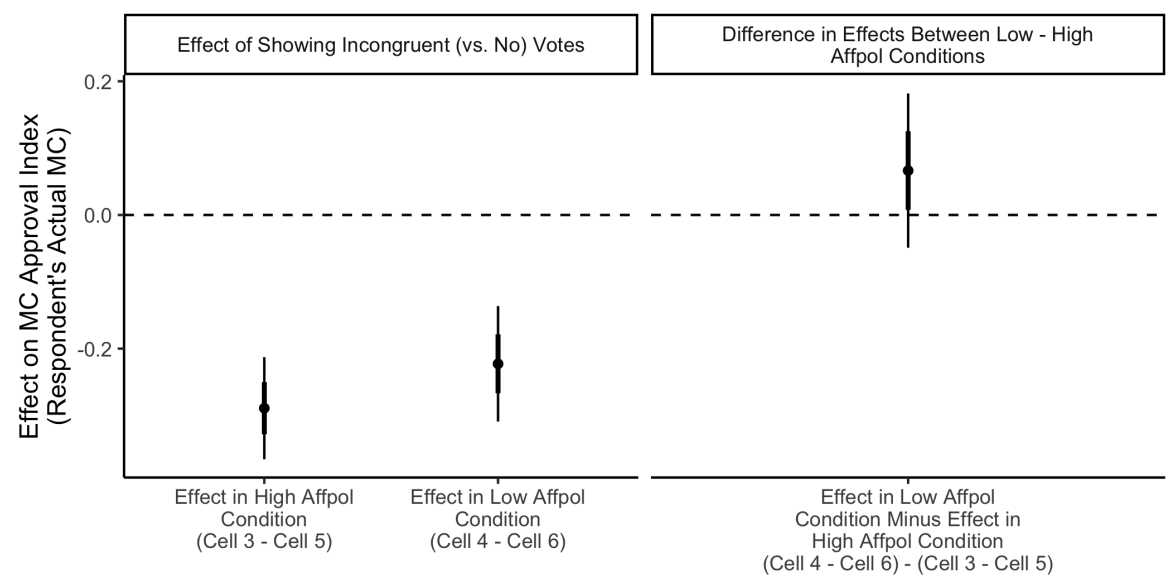

(c) Survey 3: Effect of Showing Congruent (vs. Not Showing) Votes

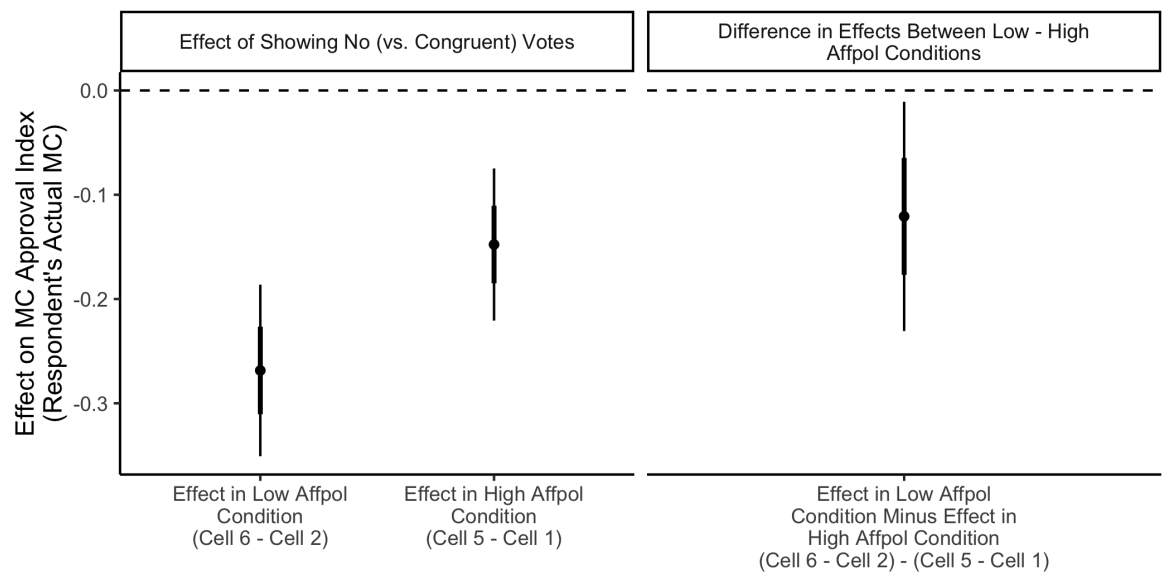

Notes: Cells refer to cells in Table 2. See Figure A1 for condition means and Figure A2 for robustness (e.g., effects split by whether MC is copartisan or outpartisan). Point estimates are surrounded by one standard error (thick tails) and $95 \%$ confidence intervals (thin tails). Point estimates are from multivariate regressions controlling for pre-registered covariates. 
decreased. The difference in these two effects between the high and low affective polarization condition is $d=0.13$; in the low affective polarization condition, respondents react if anything slightly less negatively to the incongruent votes.

In Survey 3 we replicated these results with a larger sample and included a pure control that was shown no information about how their Member of Congress voted.

The results are generally similar. Figure $5 \mathrm{~b}$ shows the effects of showing respondents incongruent votes their MC cast as compared to not showing them any votes their MC cast. As in Survey 1, we see similar effects regardless of whether respondent's level of affective polarization was reduced or increased; and, if anything, those in the decreased affective polarization condition again punished their Member of Congress less for casting incongruent votes.

When examining the effects of casting congruent votes, we do see some sign that respondents rewarded their MCs more for casting congruent votes when in the increased affective polarization condition, a statistically significant result $(p=0.03)$. However, there are several reasons to be skeptical of this result: 1) it goes in the opposite direction as the other two results, and pooling the three results yields a null result; 2) as we discuss at the end of the article, adjusting this result for multiple comparisons renders it insignificant; and 3) while insignificant in both cases, the point estimate is similar in size for participants with copartisan and outpartisan MCs (see Figure A2), inconsistent with what the standard view of how affective polarization would change reactions to congruent votes would hold (i.e., that the effect of reducing affective polarization on how people react to information about congruent votes, if real, would be driven by affective polarization leading respondents to more readily reward outpartisan politicians for congruent votes).

In summary, randomly inducing a reduction in affective polarization does not appear to encourage voters to engage in additional electoral accountability; if anything, on net, the point estimates on average indicate they engage in less of it. We also show in Figure A2 that these results hold if we look separately by whether the Member of Congress is a copartisan or outpartisan, as well as if we focus on just a survey item capturing vote intentions and not the MC 
Approval Index.10

\section{Adopting One's Party's Positions}

Several scholars have also speculated that "affective polarization" might be "associated with greater responsiveness to party cues" (Druckman et al. 2020, p. 9). This could undermine accountability if legislators could avoid responsibility for their actions by more easily persuading affectively polarized copartisan citizens of the merits of their actions. We examine this possibility with a series of additional dependent variables we asked after the MC Accountability experiments described above. After playing the trust game, learning their MC's legislative voting record, and answering questions about favorability towards their MC, we also asked respondents to again answer questions about their own issue preferences on the issues on which we showed them their MC's votes.

Are respondents less likely to adopt their party's positions on issues when their level of affective polarization is decreased? To test this, we examine whether respondents who were assigned the positive trust game experience (low affective polarization) are less likely to express post-treatment party-consistent issue preferences than those assigned to the negative trust game experience (high affective polarization). This would be expected regardless of whether respondents saw information about how a copartisan or outpartisan MC voted, as partisans could also use an outpartisan MC's vote as a negative cue. To code the dependent variable, we compute the share of issues on which the respondent gave the same position as we had previously said their MC had taken; or, in the case when respondents had outpartisan MCs, the share of issues on which they gave the opposite position.

Figure 6 shows the results. We find no substantively or statistically significant effect of exogenously reducing affective polarization on whether respondents subsequently express party-consistent issue preferences in either Survey 1 or Study 3. (This comparison corresponds with comparing issue preferences for those in Cells 2, 4, and 6 versus Cells 1, 3, and 5 of Table

\footnotetext{
${ }^{10}$ Figure $\mathrm{A} 3$ presents estimates that explore potential mechanisms.
} 
2.) The point estimates are essentially zero, and the confidence intervals are very small. We continue to find null effects when we separately examine respondents who were of the same party or different party as their Member of Congress. 11

Figure 6: Testing for Greater Receptivity to Party Cues: Effect of Inducing Affective Polarization on Party-Consistent Issue Preferences

\section{Effect of Reducing Affective Polarization on Expressing} Party-Consistent Issue Preferences

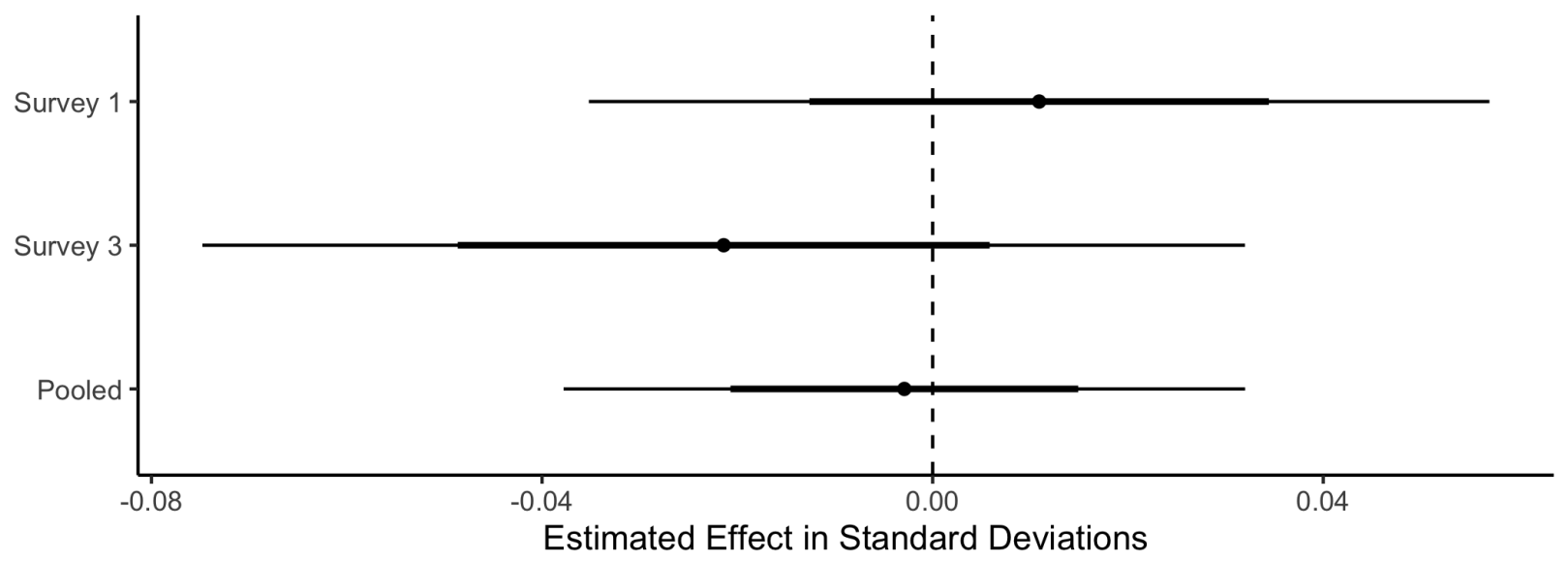

Notes: Point estimates are surrounded by one standard error (thick tails) and 95\% confidence intervals (thin tails). Point estimates are from multivariate regressions controlling for preregistered covariates.

\section{Support for Legislative Bipartisanship}

Increases in affective polarization are also often blamed for partisan gridlock. For example, Levendusky [2018, p. 59) expresses concern that "affective polarization" may "make[] governance more difficult" by discouraging legislators from compromising.

To evaluate this prediction, Survey 2 deployed a manipulation developed by Harbridge and Malhotra (2011, Study 2). The authors found two real Members of Congress, one of each party, who had each cast mostly party-line votes in one recent year but had cast a number of votes with the

\footnotetext{
${ }^{11}$ In Study 3, we also find that reducing affective polarization causes a nearly significant increase in party-consistent issue preferences when no votes are shown $(p=0.053)$, opposite from prior literature's predictions. The interaction between showing votes and the negative trust game is positive and significant $(p=0.04)$, although the effect of reducing affective polarization among those who were shown votes is not $(p=0.44)$.
} 
outparty in another recent year. The respondents are told about the Member of Congress of their party and it is randomly assigned whether they learn about the year of votes when this copartisan member cast party-line votes or often cast votes with the outparty.

After the vignette, respondents are asked whether they approve or disapprove of the job the representative is doing in Congress. Harbridge and Malhotra (2011) find that respondents on average approve of the job the representative is doing more when they learn about the year of votes where the member voted in a bipartisan manner.

We build on Harbridge and Malhotra (2011) by also randomly assigning respondents to either the positive or negative trust game experience before this vignette. If less affectively polarized voters would be more favorable towards bipartisanship, we should expect to see voters assigned to the reduced affective polarization condition additionally approve of the politician in the bipartisanship condition (or, equivalently, additionally disapprove of the politician in the partisan condition).

Figure 7 shows the results. The first coefficient shows the effect of the bipartisan condition for respondents in the high affective polarization condition. Similar to Harbridge and Malhotra (2011), we find that these respondents are more approving of the Member of Congress described as bipartisan instead of partisan. However, inconsistent with speculation in the literature on affective polarization, we do not find that respondents whose level of affective polarization has been reduced desire more bipartisan behavior. If anything, as the second coefficient shows, respondents who are assigned to be less affectively polarized reward bipartisanship less. The difference in these effects is not significant $(d=-0.11, p=0.17)$, but if anything indicates that respondents assigned to have decreased affective polarization rewarded bipartisan behavior less.

\section{Support for Democratic Norms}

We next consider whether decreasing affective polarization bolsters support for democratic norms, as considerable research speculates. To test this, in Surveys 1, 2, and 4, after respondents played the trust game, we asked a wide variety of questions using multiple approaches to probe 
Figure 7: Testing for Decreased Support for Bipartisanship in Congress

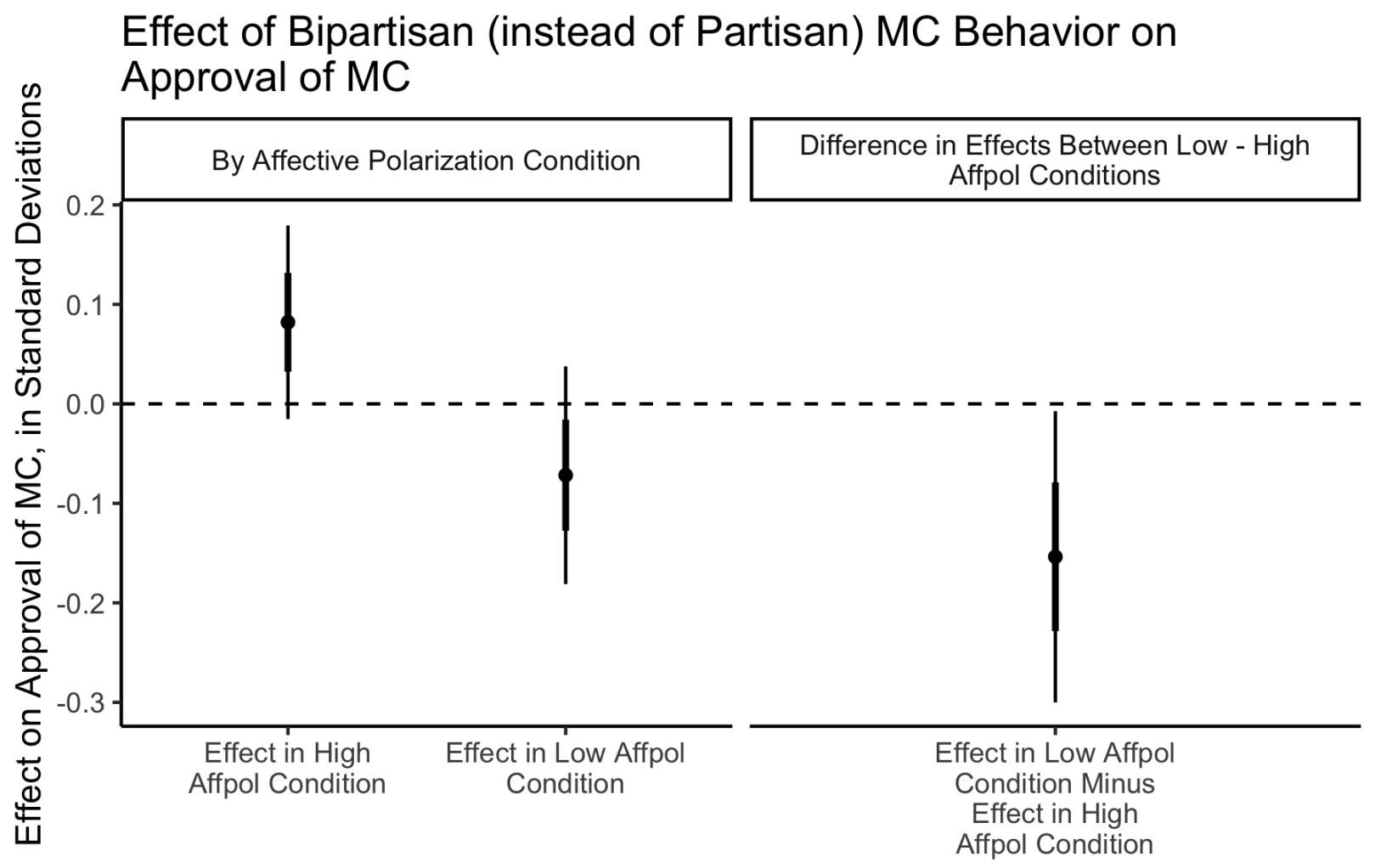

Notes: See Figure A4 for condition means. Point estimates are surrounded by one standard error (thick tails) and 95\% confidence intervals (thin tails). Point estimates are from multivariate regressions controlling for pre-registered covariates.

support for democratic norms. In particular, we asked respondents an index probing opposition to democratic norms, an index from Kalmoe and Mason (2019) on partisan violence justification, and several vignettes from Lelkes and Westwood (2017) asking respondents about specific cases of a breach in democratic norms. See Online Appendix D.4 and Table A2 for full question wording and ordering.

We estimate the effect of reducing affective polarization on these norms indices and vignettes by regressing each outcome on an indicator for the randomly assigned positive trust game experience and pre-registered pre-treatment covariates. We code the direction of all the items such that higher values correspond with greater opposition to norms, harsher actions towards 
outpartisans, and leniency towards copartisans. Therefore, the literature's expectation would be for negative coefficients (i.e., reducing affective polarization would make people less likely to oppose democratic norms).

In Figure 8, we find consistently small and statistically insignificant effects on each of the norms outcomes (the only significant result, from Survey 2, did not replicate in Survey 4). We find no evidence that an exogenous decrease in affective polarization causes a downstream decrease in opposition to democratic norms. Even those estimates with the expected negative point estimates have tight confidence intervals that overlap zero. Appendix Figure A5 shows that the same pattern holds on the individual items ${ }^{12}$ Although not all the point estimates are exactly zero, the spread of the point estimates around zero is consistent with what we would expect from sampling variability.

\section{Perceptions of Objective Conditions}

The last set of outcomes we examine is whether a decrease in affective polarization leads to more accurate perceptions of objective conditions. For example, Iyengar, Sood and Lelkes (2012, p. 428) hypothesize a connection between affective polarization and "partisan bias in perceptions of economic conditions."

We test this prediction in Survey 4 by asking respondents questions about their perceptions of unemployment rates and COVID-19 deaths. After respondents completed the trust game, we reminded respondents that "Donald Trump has been President of the United States for four years," then asked them what they thought the unemployment rate was and how many Americans had died of COVID-19. If affective polarization cause differences in stated perceptions of objective conditions, we should observe differences in how respondents answer these questions depending on their trust game condition.

As expected, there were large partisan differences in stated perceptions of the number of COVID deaths and of the unemployment rate, with Republicans stating perceptions far lower, and

\footnotetext{
${ }^{12}$ Following Kingzette et al. (2021), we examined the effects on the norms scale by party and find nulls for both parties.
} 
Figure 8: Testing for Undermined Support for Democratic Norms

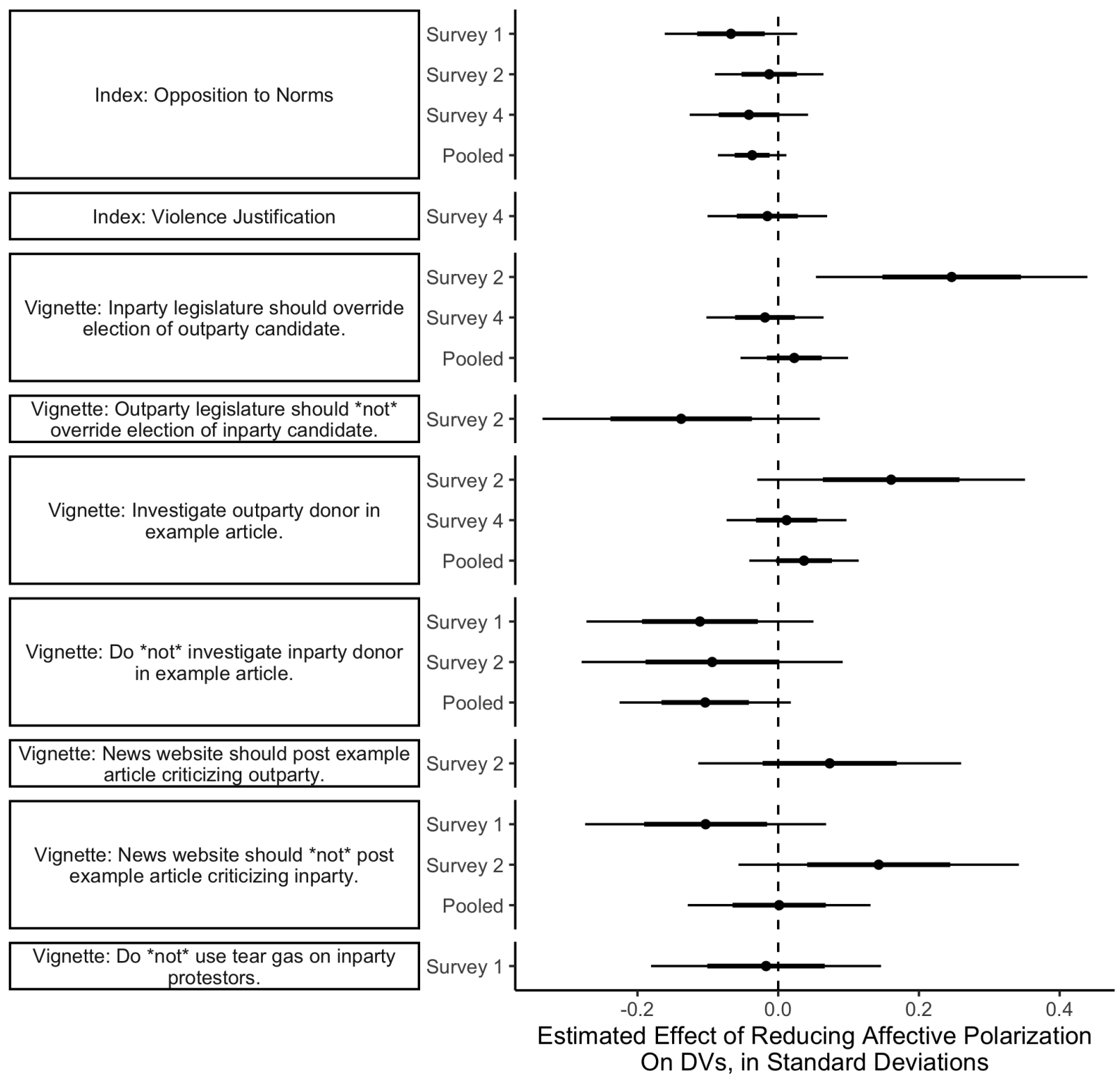

Notes: Individual items in the norms index reported in Figure A5. Individual items in the violence index reported in Figure A6. Point estimates are surrounded by one standard error (thick tails) and 95\% confidence intervals (thin tails). Point estimates are from multivariate regressions controlling for pre-registered covariates. 
Democrats stating perceptions far higher, of both. However, decreasing affective polarization did not appear to mitigate this partisan bias. Figure 9 shows these results. ${ }^{13}$

Figure 9: Testing for Increased Bias in Perceptions of Objective Conditions

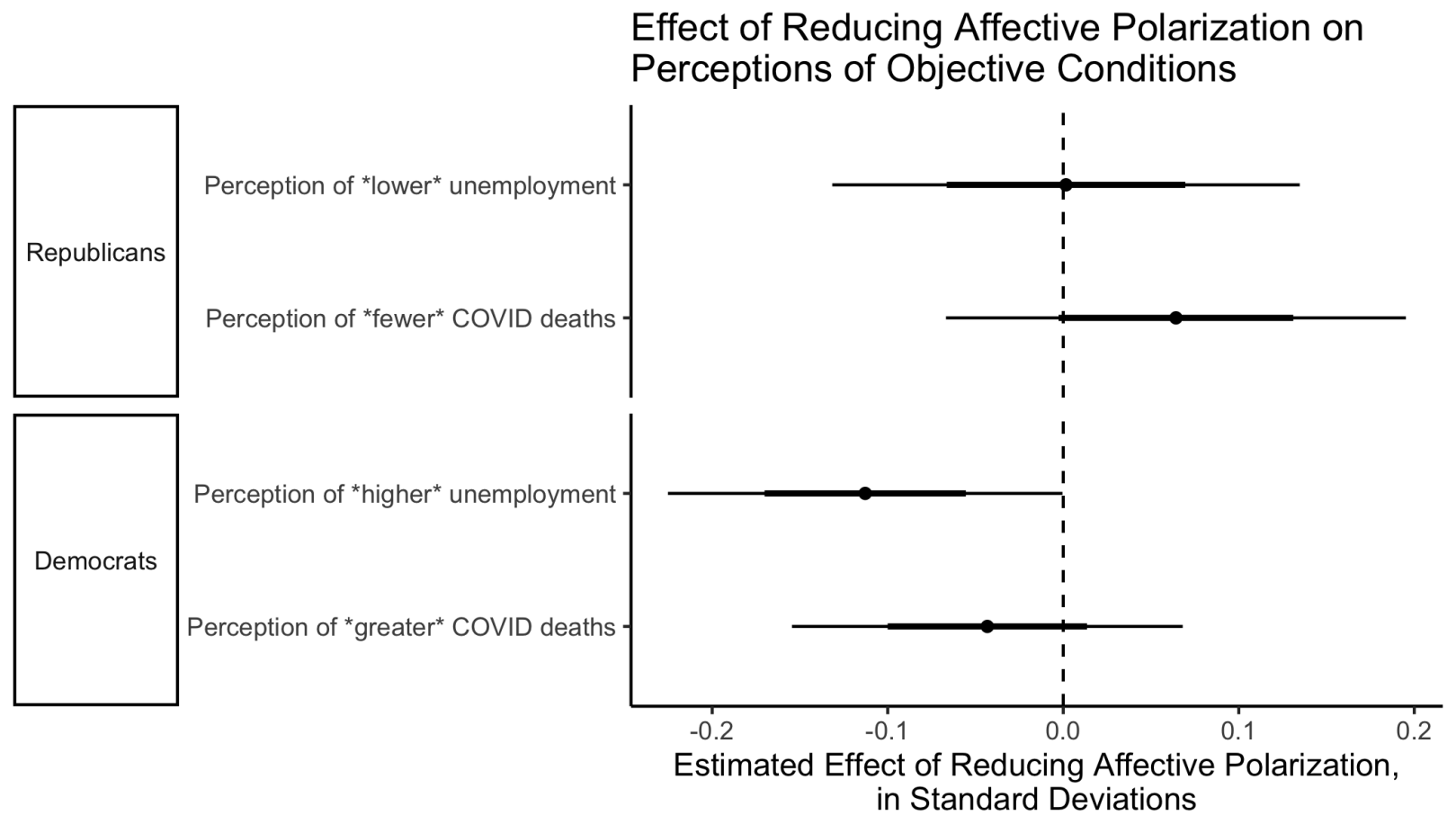

Notes: Point estimates are surrounded by one standard error (thick tails) and $95 \%$ confidence intervals (thin tails). Point estimates are from multivariate regressions controlling for preregistered covariates.

\section{Alternative Explanations and Robustness Checks: Survey 5, Alternative Manipulations of Affective Polarization}

Some may wonder whether the downstream effects of the changes in measured affective polarization the trust game produced are informative about the downstream effects of other interventions that would change affective polarization. Might reducing affective polarization in other ways have salutary downstream effects even though we did not find them with this

\footnotetext{
${ }^{13}$ The result that Democrats in the high affective polarization condition say they perceive higher levels of unemployment just meets the conventional threshold for statistical significance $(p=0.03)$ and does not survive adjusting for multiple comparisons.
} 
manipulation? This concern can be expressed more precisely in terms of four specific concerns, each of which we help address with a final pre-registered survey, Survey 5.

The first concern is that the trust game and other interventions might manipulate affective polarization among different populations (i.e., different compliers). Our evidence so far does not support this concern: Table A1 shows that the trust game appears to have successfully manipulated affective polarization broadly, at every level of the 7-point partisan identification scale. However, Survey 5 addresses this concern further.

The second concern regards the exclusion restriction: perhaps a positive experience in the trust game also affected constructs other than affective polarization and the effects of these other constructs cancelled out the salutary effects of reducing affective polarization. For this to have occurred, these other constructs would need to have undermined norms and accountability to an extent that exactly offset the salutary effects of reducing affective polarization. This seems doubtful; however, Survey 5 again addresses this concern further.

Third, one could be concerned that the trust game affected measured affective polarization but not actual affective polarization-i.e., perhaps the trust game induced non-random measurement error that led respondents to answer the survey questions measuring affective polarization differently even though actual affective polarization did not change. We did find downstream consequences of affective polarization for general social distance items (see Figure 3), and Westwood and Peterson (2020) also found downstream predicted effects of this manipulation on racial affect, suggesting this is unlikely. However, Survey 5 again addresses this further.

Fourth, an obtrusive manipulation such as the trust game may raise respondent suspicion and produce demand. For this to have led to null results, a positive trust game experience would need to have led respondents to misrepresent their attitudes in a manner consistent with less accountability and being more anti-democratic, cancelling out the salutary effects of the reduced affective polarization. Or, alternatively, it could be that the trust game did not actually manipulate affective polarization at all, but that respondents thought researchers wanted to see this occur. 
Survey 5 again addresses this further.

Survey 5 helps further address these concerns by examining the downstream effects of three additional approaches for manipulating affective polarization ${ }^{14}$ Ahler and Sood's (2018) intervention reduces affective polarization by correcting misperceptions about the demographic composition of outpartisans. Lees and Cikara's (2020) intervention alters perceptions of outpartisans' political views on a policy issue. Finally, Levendusky's (2020) intervention prompts individuals to ruminate about an outpartisan friend. Survey 5 implements all three of these interventions. These interventions complement the trust game by manipulating affective polarization in three conceptually distinct manners.

Due to space constraints, we report Survey 5 in full in Appendix F and only discuss the main points here.

In June 2021, $N=2,504$ respondents to the online survey vendor Dynata were randomly assigned to control, one of the three manipulations above, or the negative trust game manipulation. Survey respondents needed to meet the same eligibility criteria as Surveys 1-4.

Replicating the results reported by Ahler and Sood (2018), Lees and Cikara (2020), and Levendusky (2020), we report in Figure 10 that all three of these interventions reduced affective polarization by a statistically significant and similar degree compared to control. The three new interventions reduce affective polarization by 0.22 standard deviations on average $(t=-4.54$, $p<0.001$ ), or 7.4 degrees. This is about half the size of the effect of the trust game manipulation, but still meaningful.

Given the smaller "first stage" effect of these manipulations on affective polarization, Survey 5 focused on downstream outcomes in the norms and social distance domains; these only required examining differences of means (whereas the other outcome areas rely on interaction terms, which requires four times as much data to estimate with the same precision).

\footnotetext{
${ }^{14}$ This was inspired by reviewer comments and by a working paper that appeared subsequent to our initial draft of this article, Voelkel et al. (2021), which drew our attention to Lees and Cikara's (2020) manipulation, which we use in Survey 5 .
} 
Figure 10: Survey 5: Manipulation Checks

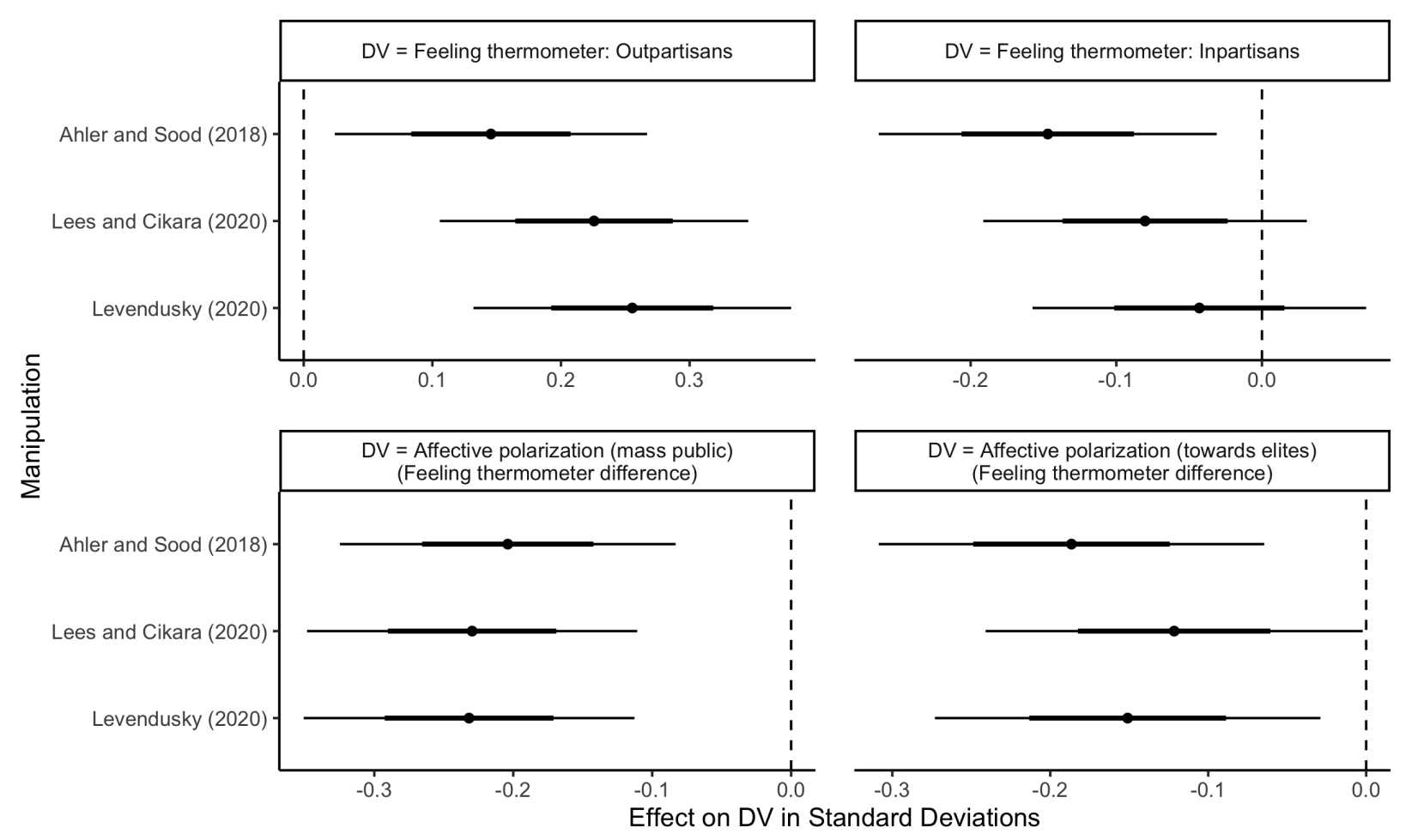

Notes: Point estimates are from multivariate regressions controlling for pre-registered covariates.

Figure 11 reports the results of Survey 5 on downstream political outcomes, estimating the effect of receiving any of the three new interventions versus the control group. We see consistent null results on political outcomes, as in Surveys 1-4. Appendix Figure A8 shows that the results are similar for all three individual interventions.

These results can help assuage concerns that our findings were particular to the trust game manipulation. Three additional, conceptually distinct approaches for reducing affective polarization produced similar results, and are unlikely to all have unusual compliers, violate the exclusion restriction in the same manner, or produce the same pattern of non-random measurement error.

As elaborated in Appendix F.2, Survey 5 also helps address the potential for demand effects by using interventions that are less overt, using additional distractor questions, and finding no signs 
Figure 11: Survey 5 Results: Average Effects of Alternative Approaches for Manipulating Affective Polarization

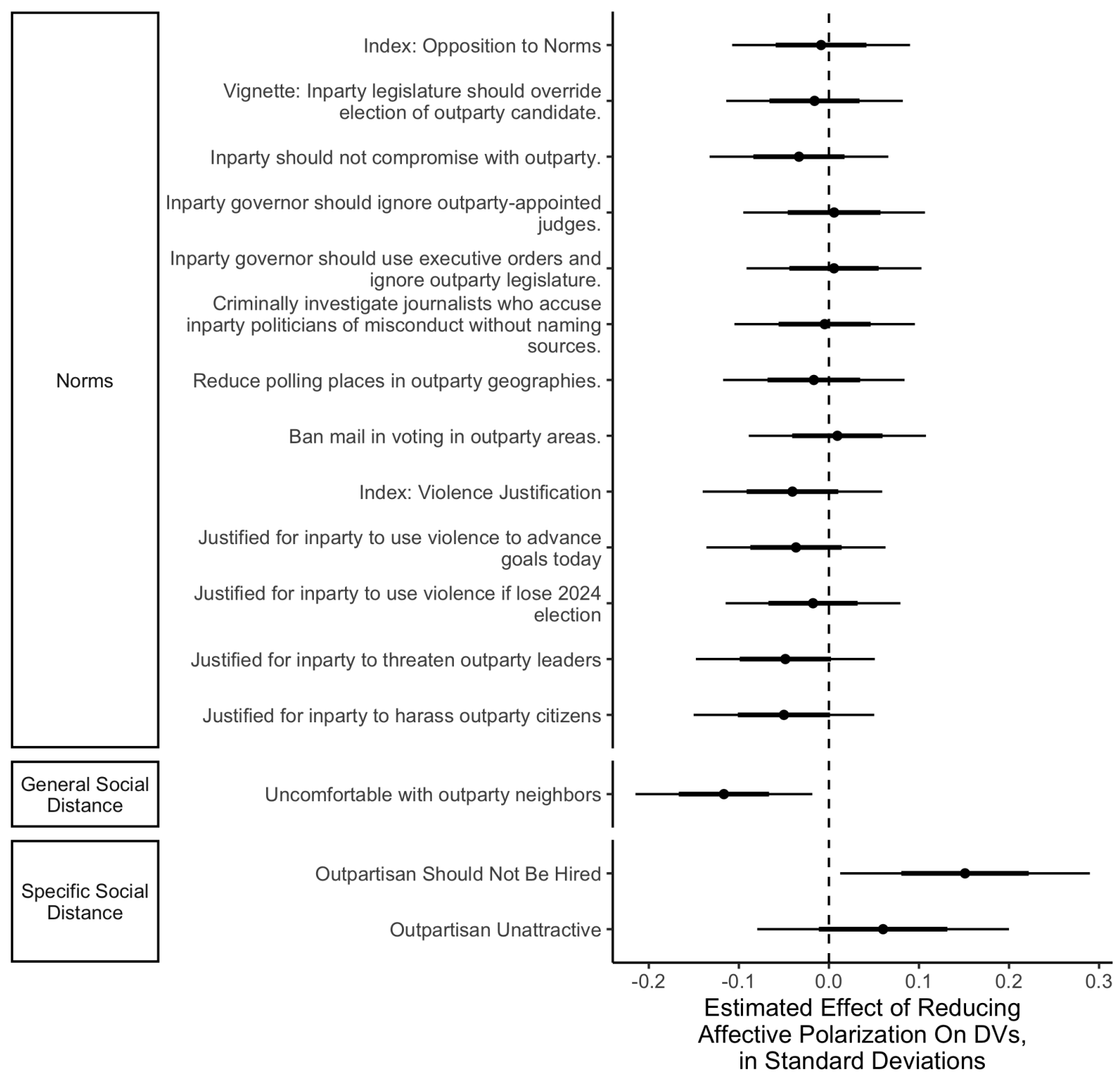

Notes: Point estimates are from multivariate regressions controlling for pre-registered covariates. Figure A8 shows that the results are similar for all three individual interventions.

of demand on open ended questions probing respondent's perceptions of the study's purpose.

Finally, consistent with Survey 5's evidence, recent papers by Voelkel et al. (2021) and Santoro and Broockman (2021), which appeared after and build on this article, also present 
interventions that reduce affective polarization but find no downstream impacts on a variety of political outcomes. Together our evidence and these papers present a total of six interventions to reduce affective polarization, none of which have downstream consequences for a wide variety of political outcomes. This bolsters confidence that this article's results are not a false negative or an artifact of the questions we asked.

By contrast, we again see that the interventions reduced discomfort with having outpartisan neighbors, consistent with a decrease in general social distance. However, as discussed further in Appendix F, we also examined effects on two new more specific social distance items. We do not find the anticipated decreases for these specific social distance items. Consistent with our argument, this suggests that the causal effects of affective polarization even in the social distance area may not be robust to more specific questions and may only appear for more abstract survey questions where trade-offs and other dimensions to judge do not exist. We return to this issue in the discussion section.

\section{Summarizing the Results}

We have presented many estimates of the downstream effects of reducing affective polarization across many outcomes and relied on multiple, conceptually distinct approaches for manipulating affective polarization. Contrary to the expectation of a great deal of literature, nearly all of our findings were null. To summarize our results and illustrate the consistency of our null findings, we computed the $t$-statistic associated with every result about the downstream effects of reducing affective polarization shown or discussed in the article. In Online Appendix G, we show that the distribution of $t$-statistics in the five political outcome areas are consistent with null results: out of the $75 t$-statistics across these five areas, only 3 are large enough to just surpass statistical significance at the conventional level, in line with what would be expected by chance. Moreover each of these 3 statistically significant results are also accompanied by other clearly null results on either replications or closely related outcomes or tests, consistent with them being false positives. These consistently null results cannot be explained by limited statistical power. Not only was 
our main manipulation of affective polarization powerful (equal to approximately three decades of increased affective polarization) and our sample sizes large, we would expect to observe a larger proportion of statistically significant $t$-statistics were the null hypothesis wrong.

\section{Comparing Experimental and Endogenous Estimates}

The null results our experimental approach surfaced stand in contrast to large and positive-but misleading-results one would reach without it. In particular, for every result we discussed or presented in the article, we computed (1) the experimental estimate we show in the article (on standardized versions of each outcome) to (2) a non-causal endogenous estimate, computed by estimating the observational relationship between the difference in affective polarization equal to the size our experimental treatment created and each standardized outcome. This allows us to compare endogenous estimates of the form that the existing literature would compute to our causal estimates on the same scale. These results are presented in Online Appendix $H$. These results provide evidence for the alternative relationship between affective polarization and downstream political choices we hypothesized in Figure 1b. Affective polarization correlates with a number of other constructs, including the intensity of partisanship, policy preferences, and many more, perhaps as it may summarize these considerations. However, this means they represent omitted variables that inflate the correlation between affective polarization and downstream choices. This makes it difficult, if not impossible, for analysts to isolate the downstream causal effects of affective polarization from the effects of these other considerations in observational data, and very likely to overestimate them.

\section{Discussion}

Across many democratic societies, citizens express increasing dislike for opposing political parties relative to their own. Scholars routinely speculate that this affective polarization has adverse downstream consequences for interpersonal and political decisions (see Table 11 for review). However, little theory or data has rigorously considered these concerns. 
Returning to foundational theories of the role of affect in judgment, we argued that we should not expect affective polarization to influence all judgments somewhat related to party, but principally those judgments where individuals do not have other, more relevant considerations they would more readily call to mind. Moreover, we pointed out that, for affective polarization to alter judgments, it creates a trade-off: individuals must give up whatever it is they preferred about the choice that affective polarization made them abandon.

For abstract survey questions about interpersonal topics, we argued that affective polarization may be more likely to have an impact: there are no trade-offs, and few other dimensions are available for respondents to rely on. However, when it comes to political judgments, our argument suggested greater skepticism—and we found no evidence that exogenously manipulating affective polarization had downstream consequences. We found these nulls across five issue areas where we leveraged a number of measurement approaches and multiple approaches for manipulating affective polarization.

\section{Limitations}

Our research is the first to rigorously examine the downstream consequences of affective polarization, but it should not be the last; our research has several important limitations.

One limitation is that we only examine the immediate effects of affective polarization on outcomes that can be measured in a survey. It is possible that there are long-run or indirect effects that our design cannot measure. For example, if affective polarization reduces interactions with outpartisans and conversations with outpartisans expose people to rationales for the other party's policies, affective polarization could have indirect effects on people's political attitudes even if it does not have direct effects on them. This is worthy of future study.

In addition, while we tested for downstream effects on the outcomes most commonly named in the literature, future research can use the paradigms we present to test for effects on additional outcomes, including evaluations of higher-profile politicians or behavioral measures. 
A third limitation is that the manipulations primarily reduced outpartisan dislike rather than inpartisan support. This is consistent with what has driven the increase in affective polarization over time (i.e., increasing dislike for the outparty Iyengar, Sood and Lelkes 2012). Manipulating inpartisan support is therefore another avenue for future research. Future research could also examine the downstream effects of further increasing affective polarization, although this may raise ethical concerns.

\section{Implications}

Our results suggest several implications for future research and for those seeking to improve American democracy.

First, our work suggests that future research on the political implications of affective polarization may wish to further examine the robustness of the downstream effects of affective polarization in the interpersonal domain. Although we found evidence of downstream effects on abstract interpersonal items, Survey 5 found that these effects did not manifest when we asked respondents to make judgments about specific individuals. It is possible that this finding speaks to a broader distinction between affective polarization's downstream effects for specific judgments versus abstract survey questions-perhaps because specific judgments involve trade-offs that answers to abstract survey questions usually do not. Or perhaps affective polarization as measured in surveys is exaggerated? Our work is too preliminary to form firm conclusions on this point, and we plan to take this question up in future research.

Second, insofar as we find some evidence of downstream effects in the social domain, future research may wish to more closely examine those areas where the interpersonal domain and the political domain are inherently linked. This could be a fruitful and important area for study that holds important normative implications. For example, we found that reducing affective polarization made individuals state that they were more willing to discuss politics with outpartisan citizens-deliberation which, if it were to occur, could have consequences our work 
was not able to measure.

Finally, and most significantly, our results have implications for efforts to improve democracy. There are a number of hypotheses for what is behind negative trends in democratic societies such as the decline in ticket splitting or the decay of certain democratic norms. In order to reverse these trends, researchers must build an understanding of which factors are contributing to these trends and which are not. We see our research as a part of this effort. It suggests that attempting to reduce affective polarization — although potentially valuable for many reasons - may not be the most effective way to reverse these trends. Other approaches, such as reducing underestimation of outpartisans' support for democratic norms (Braley et al.|2021), may be more promising. Our findings also in no way end the conversation about the potential political impacts of affective polarization on the functioning of American democracy, but rather represent a step towards more focused theorizing and rigorous empirical study of this important topic. Moreover, our findings in no way discount the role of other features of partisanship, such as partisan identity (which is distinct from affect), in potentially contributing to negative trends in democracy. Indeed, in casting doubt on the role of affective polarization, our work underscores the need to attend to these other possibilities.

\section{References}

Ahler, Douglas J. and Gaurav Sood. 2018. "The parties in our heads: Misperceptions about party composition and their consequences." Journal of Politics 80(3):964-981.

Becker, Gary S. 1957. The Economics of Discrimination. Chicago: University of Chicago Press.

Braley, Alia, Gabriel S. Lenz, Dhaval Adjodah, Hossein Rahnama and Alex Pentland. 2021. "The Subversion Dilemma: Why Voters Who Cherish Democracy Vote it Away." Available at http s://osf.io/my987.

Druckman, James N. and Matthew S. Levendusky. 2019. "What do we measure when we measure 
affective polarization?" Public Opinion Quarterly 83(1):114-122.

Druckman, James N., Samara Klar, Yanna Krupnikov, Matthew Levendusky and John Barry Ryan. 2020. "How Affective Polarization Shapes Americans' Political Beliefs: A Study of Response to the COVID-19 Pandemic." Journal of Experimental Political Science pp. 1-12.

Finkel, Eli J. et al. 2020. “Political sectarianism in America.” Science 370(6516):533-536.

Freeder, Sean. 2020. "It's No Longer the Economy, Stupid: Selective Perception and Attribution of Economic Outcomes." Available at https://seanfreeder.files.wordpress.co $\mathrm{m} / 2020 / 12 /$ paper_economy.pdf.

Graham, Matthew H and Milan W Svolik. 2020. "Democracy in America? Partisanship, Polarization, and the Robustness of Support for Democracy in the United States." American Political Science Review 114(2):392-409.

Harbridge, Laurel and Neil Malhotra. 2011. "Electoral incentives and partisan conflict in Congress: Evidence from survey experiments." American Journal of Political Science 55(3):494-510.

Iyengar, Shanto, Gaurav Sood and Yphtach Lelkes. 2012. "Affect, Not Ideology: A Social Identity Perspective on Polarization." Public Opinion Quarterly 76(3):405-431.

Iyengar, Shanto and Masha Krupenkin. 2018. "Partisanship as social identity; Implications for the study of party polarization." The Forum 16(1):23-45.

Iyengar, Shanto, Yphtach Lelkes, Matthew Levendusky, Neil Malhotra and Sean J. Westwood. 2019. "The origins and consequences of affective polarization in the United States." Annual Review of Political Science 22:129-146.

Kalmoe, Nathan P. and Lilliana Mason. 2019. "Lethal mass partisanship: Prevalence, correlates, and electoral contingencies." Available at https://www.dannyhayes.org/uploads 
/6/9/8/5/69858539/kalmoe__mason_ncapsa_2019_-_lethal_partisanshi p_-_final_lmedit.pdf.

Kingzette, Jon, James N. Druckman, Samara Klar, Yanna Krupnikov, Matthew Levendusky and John B. Ryan. 2021. "How Affective Polarization Undermines Support for Democratic Norms." Public Opinion Quarterly 85(2):663-677.

Lees, Jeffrey and Mina Cikara. 2020. "Inaccurate group meta-perceptions drive negative out-group attributions in competitive contexts.” Nature Human Behaviour 4(3):279-286.

Lelkes, Yphtach and Sean J. Westwood. 2017. “The limits of partisan prejudice." Journal of Politics 79(2):485-501.

Levendusky, Matthew S. 2018. "Americans, not partisans: Can priming American national identity reduce affective polarization?" Journal of Politics 80(1):59-70.

Levendusky, Matthew S. 2020. "Our common bonds: Using what Americans share to help bridge the partisan divide." Unpublished Manuscript .

Little, Andrew T., Keith Schnakenberg and Ian R. Turner. 2022. "Motivated reasoning and democratic accountability." American Political Science Review .

McCoy, Jennifer and Murat Somer. 2019. "Toward a theory of pernicious polarization and how it harms democracies: Comparative evidence and possible remedies." The Annals of the American Academy of Political and Social Science 681(1):234-271.

Ottati, Victor C. and Robert S. Wyer. 1993. Affect and Political Judgment. In Explorations in political psychology, ed. Shanto Iyengar and William J. McGuire. Duke pp. 296-316.

Pierson, Paul and Eric Schickler. 2020. “Madison's Constitution Under Stress: A Developmental Analysis of Political Polarization.” Annual Review of Political Science 23:37-58. 
Santoro, Erik and David Broockman. 2021. "The short-term, circumscribed, and conditional effects of cross-partisan conversation.” Available at https: / / osf . io/km3q2.

Slovic, Paul, Melissa L. Finucane, Ellen Peters and Donald G. MacGregor. 2002. The affect heuristic. In Heuristics and Biases: The Psychology of Intuitive Judgment, ed. Thomas Gilovich, Dale Griffin and Daniel Kahneman. Cambridge.

Voelkel, Jan G. et al. 2021. "Interventions Reducing Affective Polarization Do Not Improve AntiDemocratic Attitudes." Available at https://osf.io/preprints/7evmp/.

Westwood, Sean J. and Erik Peterson. 2020. "The Inseparability of Race and Partisanship in the United States." Political Behavior pp. 1-23. 


\section{Online Appendix}

\section{Online Appendix Page Count: 25/25 allowed}

\section{Contents}

\begin{tabular}{|l|l}
\hline A Ethical Statement & 2
\end{tabular}

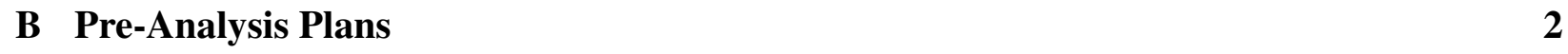

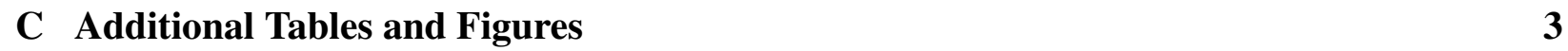

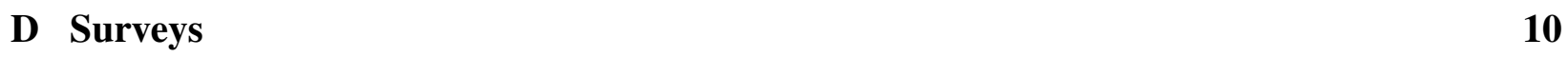

D.1 Survey Contents and Order . . . . . . . . . . . . . . . . . . 10

D.2 Survey Demographics and Representativeness . . . . . . . . . . . . . . . 11

D.3 Attrition . . . . . . . . . . . . . . . . . . . 12

D.4 Survey Questions . . . . . . . . . . . . . . . . . . . . 14

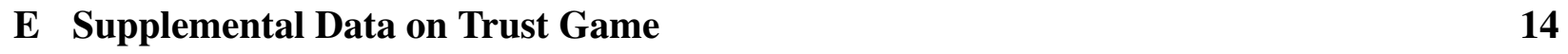

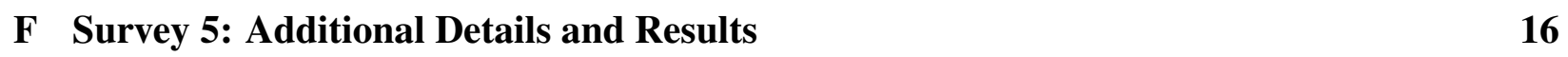

F.1 Results . . . . . . . . . . . . . . . . . . . . . . . 17

F.2 Testing for Demand Effects . . . . . . . . . . . . . . . . 20

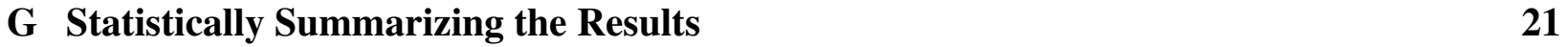

\begin{tabular}{|ll}
\hline H Comparing Experimental and Endogenous Estimates & 23
\end{tabular}

\begin{tabular}{|l|l|}
\hline I References for Online Appendix & 25
\end{tabular} 


\section{A Ethical Statement}

Surveys were IRB approved and we obtained voluntary and informed consent from all subjects. Subjects were aware they were taking part in a research study. Our research design required the use of deception. Participants were aware that deception might be used and we debriefed all subjects immediately following the conclusion of the survey. The survey vendor offered a predetermined, fair level of compensation to participants based on the length of the survey.

\section{B Pre-Analysis Plans}

To access fully anonymized versions of the pre-analysis plans, please visit:

- Survey 1: https://osf.io/kde27/?view_only=9b450ae09e234f0ba3f719 3432170530 .

- Survey 2: https://osf.io/7ve49/?view_only=6d36403f548c43a6817e24 c079d91d30.

- Survey 3: https://osf.io/7ve49/?view_only=6d36403f548c43a6817e24 c079d91d30.

- Survey 4: https://osf.io/2ysp7/?view_only=1903af75960a4683a4238f $835 d 5 e f f 53$.

- Survey 5: https://osf.io/v3qc8/?view_only=eef037e9e32b4a 4ea 4 fed5 4befd2190f.

Across these PAPs we pre-registered 26 primary hypotheses, all of which we report in the main text or appendix of this article. 


\section{Additional Tables and Figures}

Table A1: Effect of Positive Trust Game Experience on Affective Polarization Towards Mass Public and Towards Elites, by Strength of Party ID

\begin{tabular}{lcc}
\hline & DV $=$ Outparty - Inparty & DV $=$ Outparty - Inparty \\
& Mass Public & Elite \\
& Feeling Therms & Feeling Therms \\
\hline PID = Not Strong Democrat & $-22.2^{* * *}$ & $-22.0^{* * *}$ \\
& $(2.85)$ & $(2.86)$ \\
PID = Lean Democrat & $-27.8^{* * *}$ & $-24.4^{* * *}$ \\
PID = Lean Republican & $(2.94)$ & $(2.92)$ \\
PID = Not Strong Republican & $-28.5^{* * *}$ & $-29.4^{* * *}$ \\
PID = Strong Republican & $(3.42)$ & $(3.51)$ \\
& $-29.1^{* * *}$ & $-30.8^{* * *}$ \\
Strong Democrat $\times$ Positive Game Experience & $(3.26)$ & $(3.31)$ \\
& -3.26 & -1.45 \\
Not Strong Democrat $\times$ Positive Game Experience & $(3.13)$ & $(3.07)$ \\
Lean Democrat $\times$ Positive Game Experience & $-15.7^{* * *}$ & $-9.86^{* * *}$ \\
& $(2.65)$ & $(2.88)$ \\
Lean Republican $\times$ Positive Game Experience & $-13.9^{* * *}$ & $-10.2^{* *}$ \\
Not Strong Republican $\times$ Positive Game Experience & $(3.07)$ & $(3.24)$ \\
Strong Republican $\times$ Positive Game Experience & $-10.2^{* *}$ & -4.46 \\
Constant & $(3.37)$ & $(3.42)$ \\
\hline \hline & $-10.8^{*}$ & -5.21 \\
& $(4.34)$ & $(4.59)$ \\
& $-16.5^{* * *}$ & $-11.2^{* *}$ \\
& $(3.44)$ & $(3.71)$ \\
& $-18.0^{* * *}$ & $-12.6^{* * *}$ \\
& $(3.69)$ & $(3.71)$ \\
& $52.7^{* * *}$ & $54.1^{* * *}$ \\
& $(1.76)$ & 2135 \\
\hline
\end{tabular}

Notes: The rows with interactions show the effect of a positive game experience by party ID. The rows without interactions show differences between levels of affective polarization between different partisan groups within the control (negative game experience) condition, with PID = Strong Democrat as the omitted baseline category. Data are from Survey 4, as Survey 4 was the only survey that asked about affective polarization towards elites. Standard errors in parentheses. $* p<0.05, * * p<0.01, * * * p<0.001$. 
Figure A1: Condition Means in MC Vote Choice Experiment

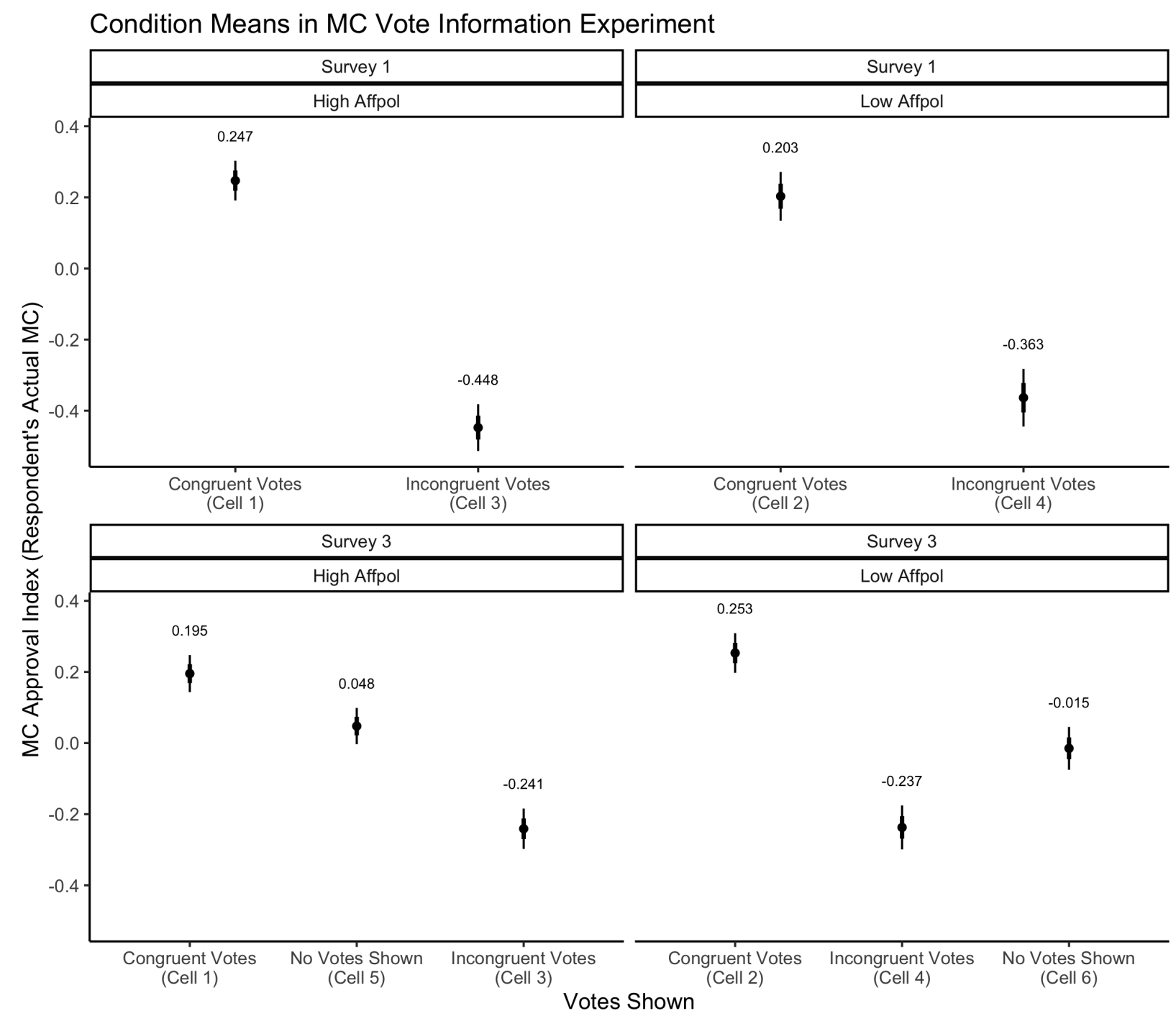

Notes: The Cell numbers in the coefficient labels refer to cells in Table 2 This Figure shows the condition means in the experiment reported in Figure 5 in the main text. Point estimates are surrounded by one standard error (thick tails) and 95\% confidence intervals (thin tails). Point estimates are predicted probabilities from multivariate regressions controlling for pre-registered covariates. 
Figure A2: Testing for Desensitization: Robustness

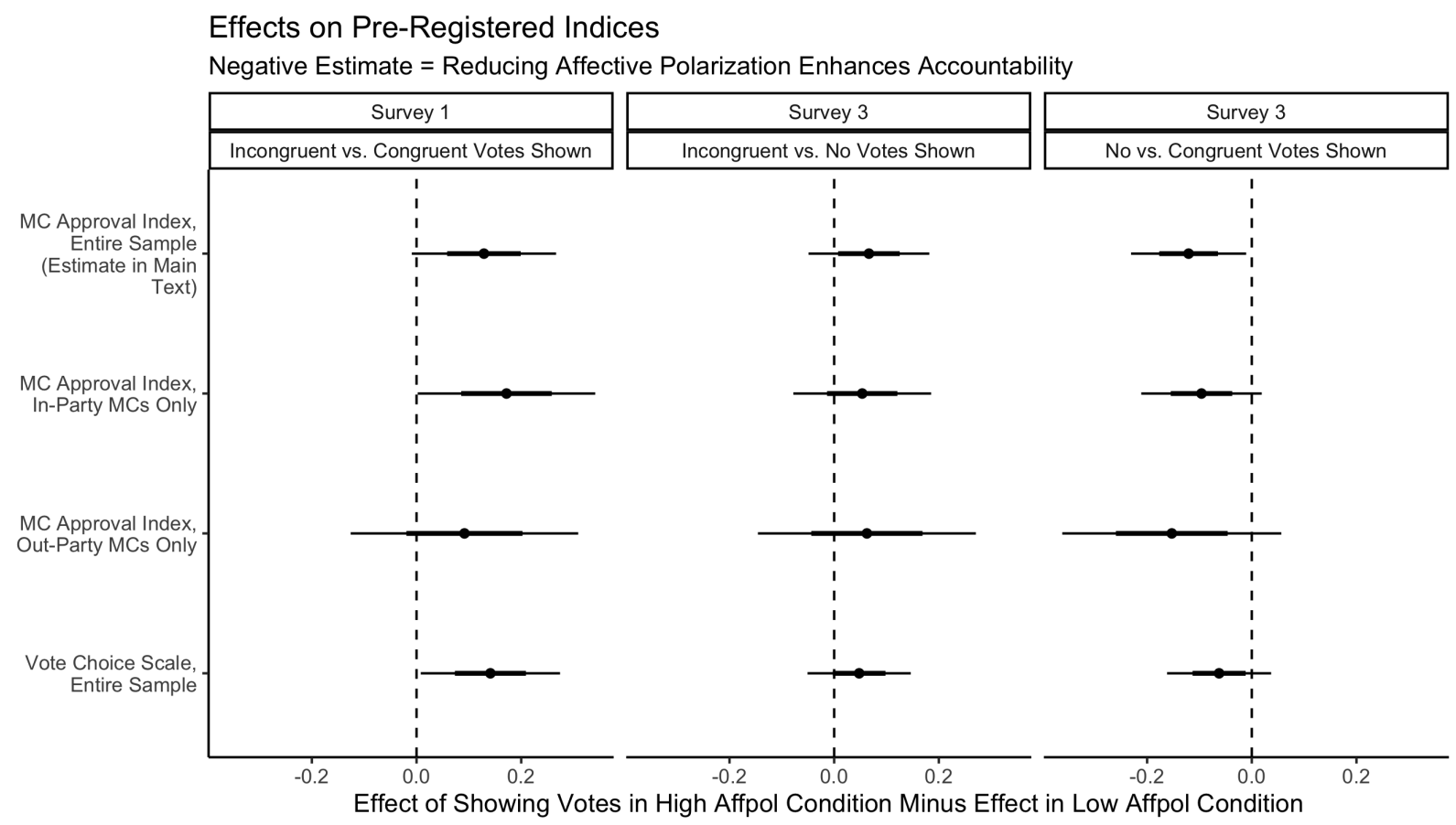

Notes: This Figure helps assess the robustness of the results reported in Figure 5 in the main text. The coefficients shown capture the interaction between the affective polarization and MC vote treatments; the first row in each panel reports the same coefficient as shown in the main text (i.e., the rightmost coefficients in each panel in Figure 5). The next two rows show the estimates for respondents with in-party MCs only and the following row the estimates for respondents with out-party MCs only, respectively. Under the hypothesis that reducing affective polarization makes individuals more willing to punish inparty MCs for incongruent votes, we should expect to see a negative coefficient in the middle "Incongruent vs. No Votes Shown" panel in the second row for "In-Party MCs Only." Under the hypothesis that reducing affective polarization makes individuals more willing to reward outparty MCs for congruent votes, we should expect to see a negative coefficient under the third panel, "No vs. Congruent Votes Shown" in the third row for "Out-Party MCs Only." The final row the results in the entire sample when using just the vote choice scale as an outcome instead of the entire MC approval index. Point estimates are surrounded by one standard error (thick tails) and 95\% confidence intervals (thin tails). Point estimates are from multivariate regressions controlling for pre-registered covariates. 
Figure A3: Testing for Desensitization: Potential Mechanisms

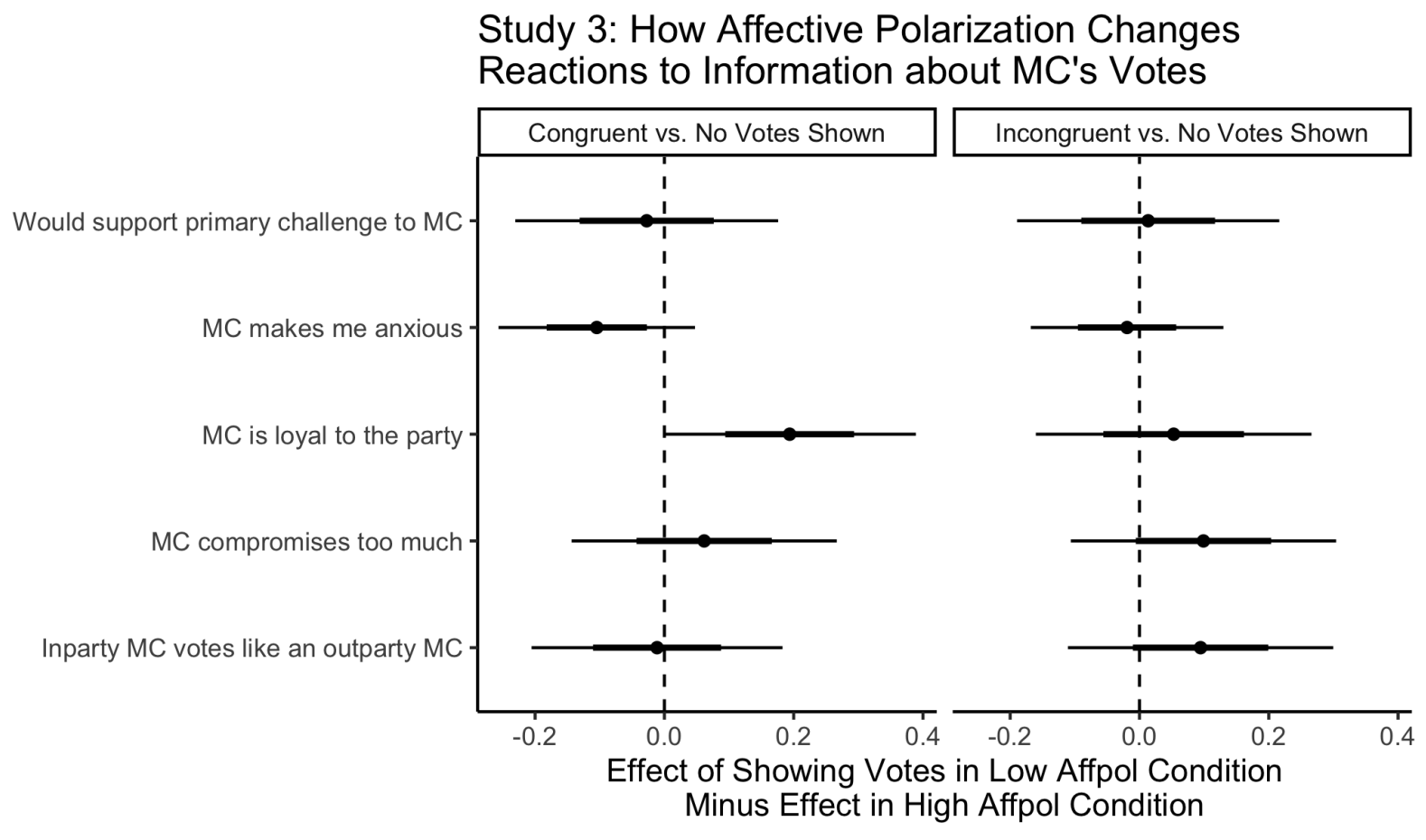

Notes: This Figure reports the difference-in-differences estimate from Figures $5 b$ and $5 c$ in the main text but on other "mechanism" items. Point estimates are surrounded by one standard error (thick tails) and 95\% confidence intervals (thin tails). Point estimates are from multivariate regressions controlling for pre-registered covariates. 
Figure A4: Condition Means in Bipartisanship Experiment

\section{Condition Means in Bipartisanship Experiment}

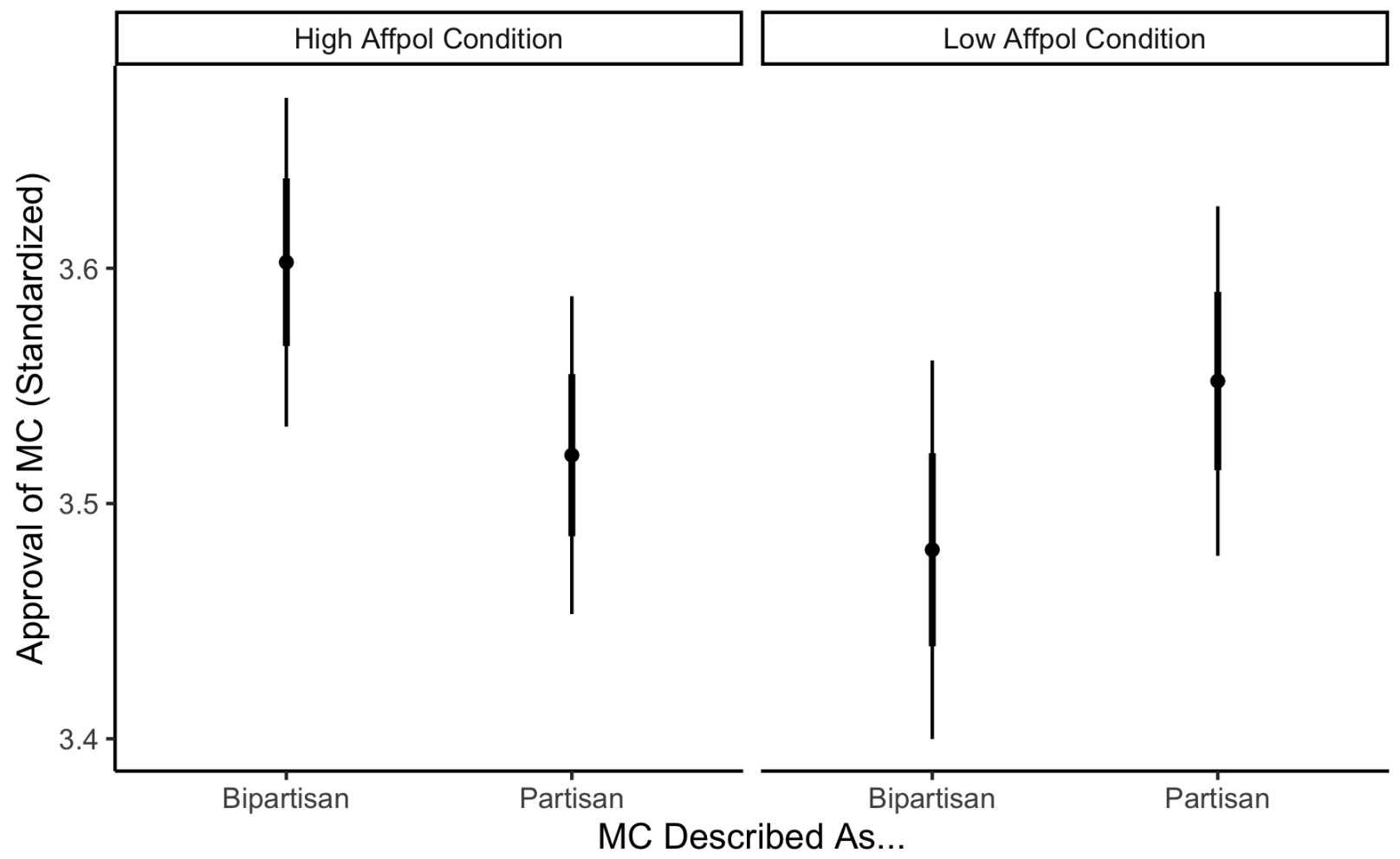

Notes: This Figure shows the condition means in the bipartisanship experiment reported in Figure 7 in the main text. Point estimates are surrounded by one standard error (thick tails) and 95\% confidence intervals (thin tails). Point estimates are from multivariate regressions controlling for pre-registered covariates. 
Figure A5: Effect of Reducing Affective Polarization on Individual Items in Norms Index

\section{Effect of Reducing Affective Polarization on Norms Items}

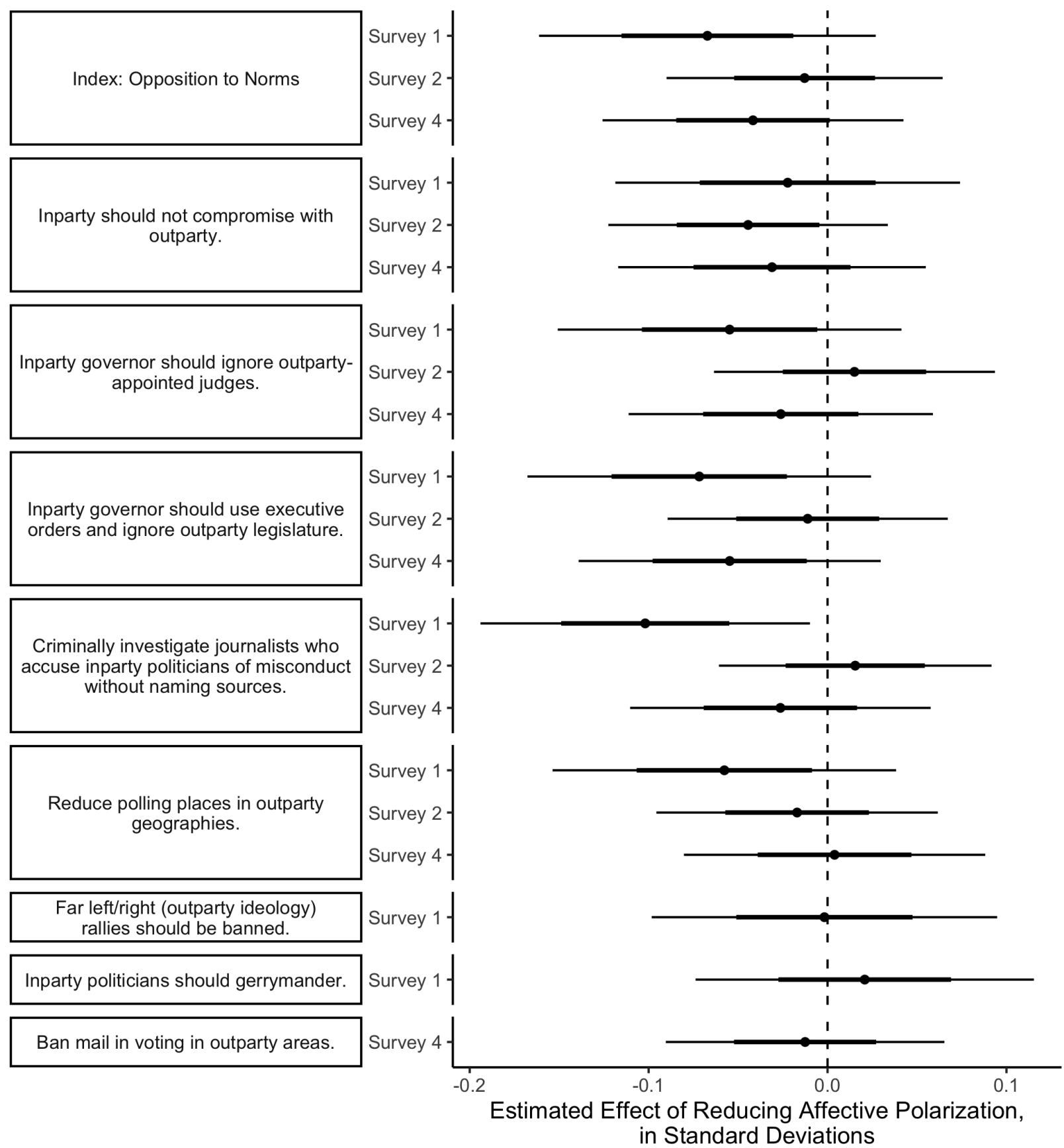

Notes: The first set of coefficients reproduces the estimates on the Norms index shown in Figure 8 in the main text. The remaining coefficients show estimates on the individual items in this index. Point estimates are surrounded by one standard error (thick tails) and 95\% confidence intervals (thin tails). Point estimates are from multivariate regressions controlling for pre-registered covariates. 
Figure A6: Effect of Reducing Affective Polarization on Individual Items in Violence Justification Index

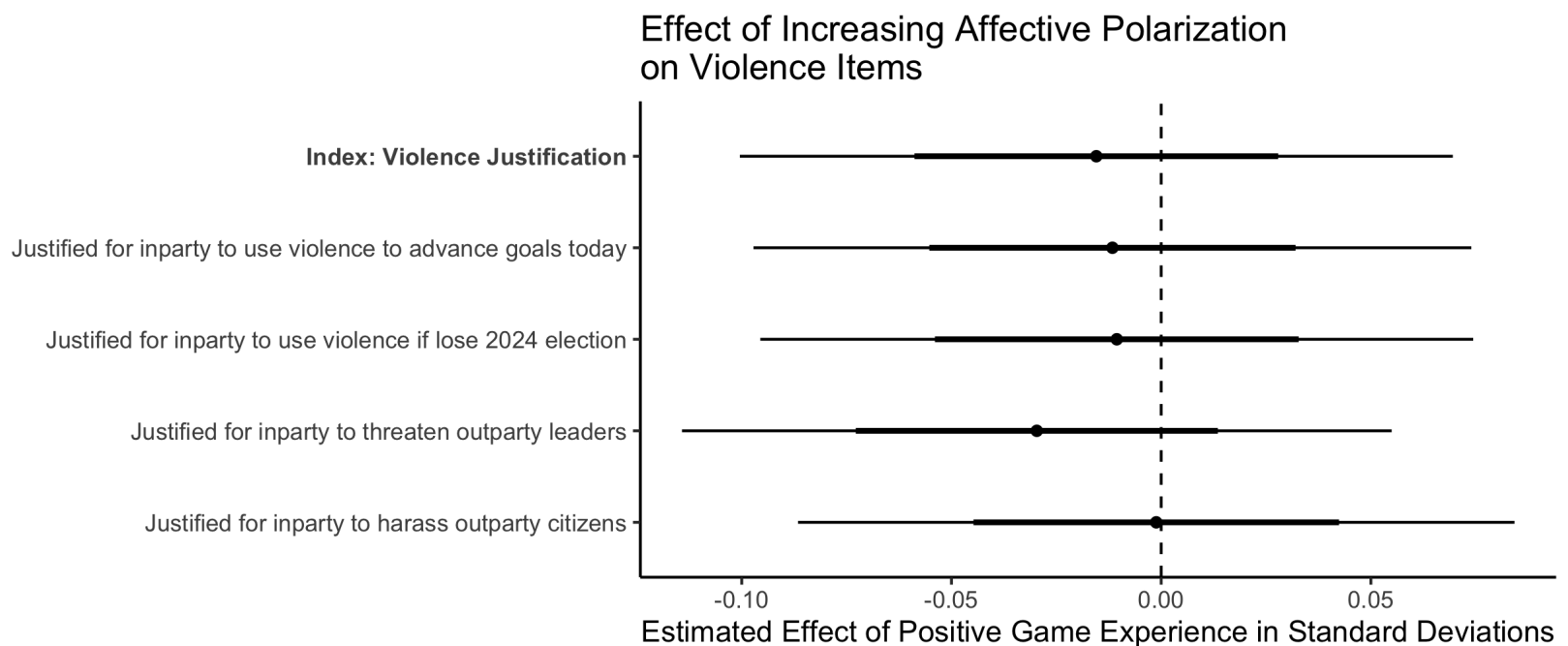

Notes: The first set of coefficients reproduces the estimates on the Violence Justification index shown in Figure 8 in the main text. The remaining coefficients show estimates on the individual items in this index. Point estimates are surrounded by one standard error (thick tails) and 95\% confidence intervals (thin tails). Point estimates are from multivariate regressions controlling for pre-registered covariates. 


\section{Surveys}

\section{D.1 Survey Contents and Order}

Table A2: Survey Contents and Order, by Survey

\begin{tabular}{|c|c|c|c|c|c|}
\hline & Survey 1 & Survey 2 & Survey 3 & Survey 4 & Survey 5 \\
\hline Demographics Battery & 1 & 1 & 1 & 1 & 1 \\
\hline $\begin{array}{l}\text { Asked Own Views on Issues } \\
\text { Pre-Treatment }\end{array}$ & 2 & & 2 & & \\
\hline Trust Game & 3 & 2 & 3 & 2 & $2^{\wedge}$ \\
\hline $\begin{array}{l}\text { Manipulation Check: } \\
\text { Treated Fairly }\end{array}$ & 4 & 3 & 4 & 3 & $3^{*}$ \\
\hline $\begin{array}{l}\text { Manipulation Check: Mass } \\
\text { Feeling Thermometers }\end{array}$ & 5 & 4 & 5 & 4 & 4 \\
\hline $\begin{array}{l}\text { Manipulation Check: } \\
\text { Salience of Partisan } \\
\text { Identity }\end{array}$ & 6 & & & & \\
\hline $\begin{array}{l}\text { Manipulation Check: Elite } \\
\text { Feeling Thermometers }\end{array}$ & & & & 5 & 5 \\
\hline Social Distance Items & & & & 6 & $6^{\star}$ \\
\hline $\begin{array}{l}\text { Shown MC Votes \& Asked } \\
\text { MC DVs }\end{array}$ & 7 & & 6 & & \\
\hline Norms Index & 8 & 5 & & $7^{\dagger}$ & $7^{\dagger}$ \\
\hline $\begin{array}{l}\text { Asked Own Views on Issues } \\
\text { Post-Treatment }\end{array}$ & 9 & & 7 & & \\
\hline Suppression Vignette & $\begin{array}{c}10^{\ddagger} \text { (In- } \\
\text { party only) }\end{array}$ & & & & \\
\hline $\begin{array}{l}\text { Corruption Tolerance } \\
\text { Vignette }\end{array}$ & $\begin{array}{c}11^{\ddagger} \text { (In- } \\
\text { party only) }\end{array}$ & $\begin{array}{c}6^{\ddagger}(\text { Party } \\
\text { randomized })\end{array}$ & & $\begin{array}{c}8^{\dagger} \text { (Out- } \\
\text { party only) }\end{array}$ & \\
\hline Antilocution Vignette & $\begin{array}{c}12^{\ddagger} \text { (In- } \\
\text { party only) }\end{array}$ & $\begin{array}{c}8^{\ddagger} \text { (Party } \\
\text { randomized })\end{array}$ & & & \\
\hline Election Override Vignette & & $\begin{array}{c}7^{\ddagger} \text { (Party } \\
\text { randomized) }\end{array}$ & & $\begin{array}{c}9^{\dagger}(\text { In- } \\
\text { party only) }\end{array}$ & $\begin{array}{c}8^{\dagger} \text { (In- } \\
\text { party only) }\end{array}$ \\
\hline Bipartisanship Vignette & & 9 & & & \\
\hline Violence Justification Index & & & & 10 & $9^{\dagger}$ \\
\hline $\begin{array}{l}\text { Perceptions of Objective } \\
\text { Conditions }\end{array}$ & & & & 11 & \\
\hline Final Demographics & 13 & 10 & 8 & 12 & 10 \\
\hline
\end{tabular}

$\ddagger$ : Respondents were randomized to just one of these vignettes.

$\wedge$ : Respondents were randomly assigned to the negative trust game, control, or one of the additional treatments designed to reduce affective polarization.

*: This was only asked for the negative trust game condition.

*: Respondents were also asked the specific social distance vignettes on outpartisan attractiveness or outpartisan worker. Respondents were randomized to just one vignette.

${ }^{\dagger}$ : The order of these items was randomized. 


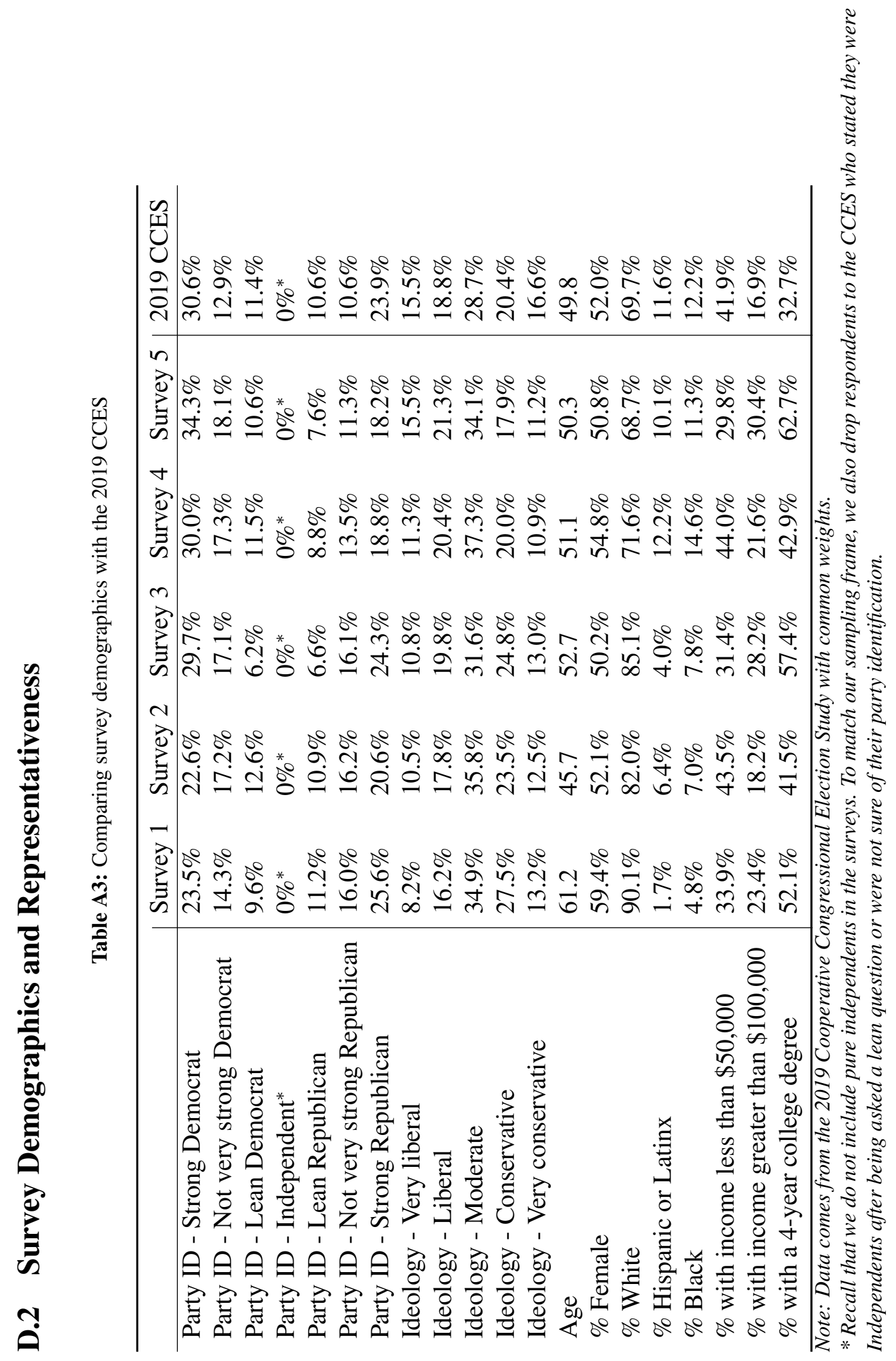


Table A3 shows the representativeness of the survey samples relative to the 2019 CCES on demographics. In addition, on Survey 5, we asked a political knowledge question to benchmark the political knowledge of samples recruited from our survey vendor with our particular approach, exclusions rule, etc. We picked a question from the 2019 Pew Research Center's American Trends Panel, Wave 57. The question asked "As you may know, presidents are chosen not by direct popular vote, but by the electoral college in which each state casts electoral votes. What determines the number of electoral votes a state has?" The response options were "The number of seats the state has in the U.S. House and Senate" (correct), "The number of voters in the state," "The number of counties in the state," "Each state has the same number of electoral votes," and "Not sure." In survey $5,37 \%$ of respondents got this question correct, versus $39 \%$ of respondents to the Pew survey.

\section{D.3 Attrition}

If subjects in different treatment conditions drop out of the survey at different rates (differential attrition), this may introduce bias into our estimated treatment effects. We find that there are statistically significant but very small differences between the share of individuals who completed the survey in different treatments in some of our surveys. Moreover, because the rates of survey completion among those assigned to treatment are extremely high and the differences between completion rates by treatment are small, we do not find that our confidence intervals grow particularly meaningfully.

In particular, in Table A4, we report survey completion rates by survey and by treatment condition. In the bottom row, we report a $p$-value from Pearson's chi-squared test to assess how likely it is that the observed differences between the treatment arms in each survey arose by chance.

From Table A4, we first observe high rates of survey completion across all treatment arms. However, we do observe some small but statistically significant differential attrition in Surveys 1, 2 , and 5 . 
Table A4: Survey Completion Rates by Treatment and Survey

\begin{tabular}{l|lllll}
\hline & Survey 1 & Survey 2 & Survey 3 & Survey 4 & Survey 5 \\
\hline Positive Game & $91.3 \%$ & $94.0 \%$ & $97.3 \%$ & $94.1 \%$ & \\
Negative Game & $96.1 \%$ & $96.5 \%$ & $98.2 \%$ & $94.5 \%$ & $97.5 \%$ \\
Control & & & & & $98.1 \%$ \\
Ahler-Sood & & & & & $95.2 \%$ \\
Lees-Cikara & & & & & $96.7 \%$ \\
Outparty Friend & & & & & $94.8 \%$ \\
\hline$p$-value & $<0.001$ & 0.003 & 0.051 & 0.664 & 0.015 \\
\hline
\end{tabular}

Table A5: 95\% Confidence Intervals of Treatment Effect on Affective Polarization Using Lee (2009) Bounds

\begin{tabular}{|c|c|c|c|c|c|c|}
\hline & & Survey 1 & Survey 2 & Survey 3 & Survey 4 & Survey 5 \\
\hline $\begin{array}{l}\text { Positive } \\
\text { Negative }\end{array}$ & vs. & {$[-0.530,-0.285]$} & {$[-0.613,-0.365]$} & {$[-0.487,-0.323]$} & $\mathrm{n} / \mathrm{a}$ & \\
\hline $\begin{array}{l}\text { Negative } \\
\text { Control }\end{array}$ & vs. & & & & & {$[-0.080,0.256]$} \\
\hline $\begin{array}{l}\text { Ahler-Sood } \\
\text { Control }\end{array}$ & vs. & & & & & {$[-0.363,0.029]$} \\
\hline $\begin{array}{l}\text { Lees-Cikara } \\
\text { Control }\end{array}$ & & & & & & {$[-0.357,-0.017]$} \\
\hline $\begin{array}{l}\text { Outparty } \\
\text { vs. Control }\end{array}$ & end & & & & & {$[-0.400,-0.016]$} \\
\hline
\end{tabular}

In order to assess whether the treatment effects we report in the main text are robust to addressing this differential attrition, in Table A5 we report 95\% confidence intervals of the effect of each treatment on our measure of affective polarization using Lee (2009) trimming bounds. Overall, we find statistically significant effects on affective polarization, even after using trimming bounds. This suggests that the effects on affective polarization are not artifacts of differential attrition.

We do not report trimming bounds for any of the downstream outcomes. Trimming bounds would only increase the width of confidence intervals that we report in the main text. The null effects that we report in the main text would therefore remain null.

We also note that prior to randomization, $48 \%$ of participants who started Survey 1 were deemed eligible because they met all criteria (informed consent, identify as a Democrat or a 
Republican (including as a leaner), pass a pre-treatment attention check, and demonstrate understanding of the trust game), $45 \%$ for Survey 2, 56\% for Survey 3, and 52\% for Survey 4. Ineligible respondents were dropped prior to randomization. Respondents were also ineligible if they had participated in an earlier survey.

\section{D.4 Survey Questions}

For full wording of the surveys and manipulations, see https://osf.io/zfy $9 \mathrm{w} /$ ?vie W_only $=44$ f370 fb5bba 4c1688b17bb1c699c684

\section{E Supplemental Data on Trust Game}

As part of a survey for a different project conducted by one of the authors, we conducted supplemental data collection using the Qualtrics Panel. In this survey, $N=249$ respondents were randomly assigned to either a control group, the negative trust game condition, or the positive trust game condition. (This stands in contrast to the data collection for Surveys 1-4 in the main text, where there was no pure control, and all respondents were assigned to either the negative or positive trust game condition.) We then assessed treatment effects on affective polarization. This survey did not measure downstream political outcomes.

Survey 5 (described in Appendix F) also had a negative trust game condition and a control condition (but no positive trust game condition).

Figure A7 shows the results. Overall, we find that the trust game manipulates affective polarization by decreasing affective polarization in the positive game experience condition relative to the negative game experience condition.

First, the left panel of Figure A7 shows that there is a null effect of the negative trust game condition on affective polarization. The first row shows the difference between the level of affective polarization in the control condition versus the negative trust game experience condition

in Survey 5; i.e., the effect of the negative trust game condition as compared to control. The second row shows the same effect in the supplemental data collection. The final row pools these 
two coefficients. The results are null in both datasets. Moreover, when pooling these two coefficients, the average effect of the negative game experience on affective polarization is 0.12 degrees, essentially exactly zero $(S E=2.01, t=0.06)$.

Figure A7: Supplemental Data on Trust Game: Effect of Positive and Negative Trust Games vs. Control

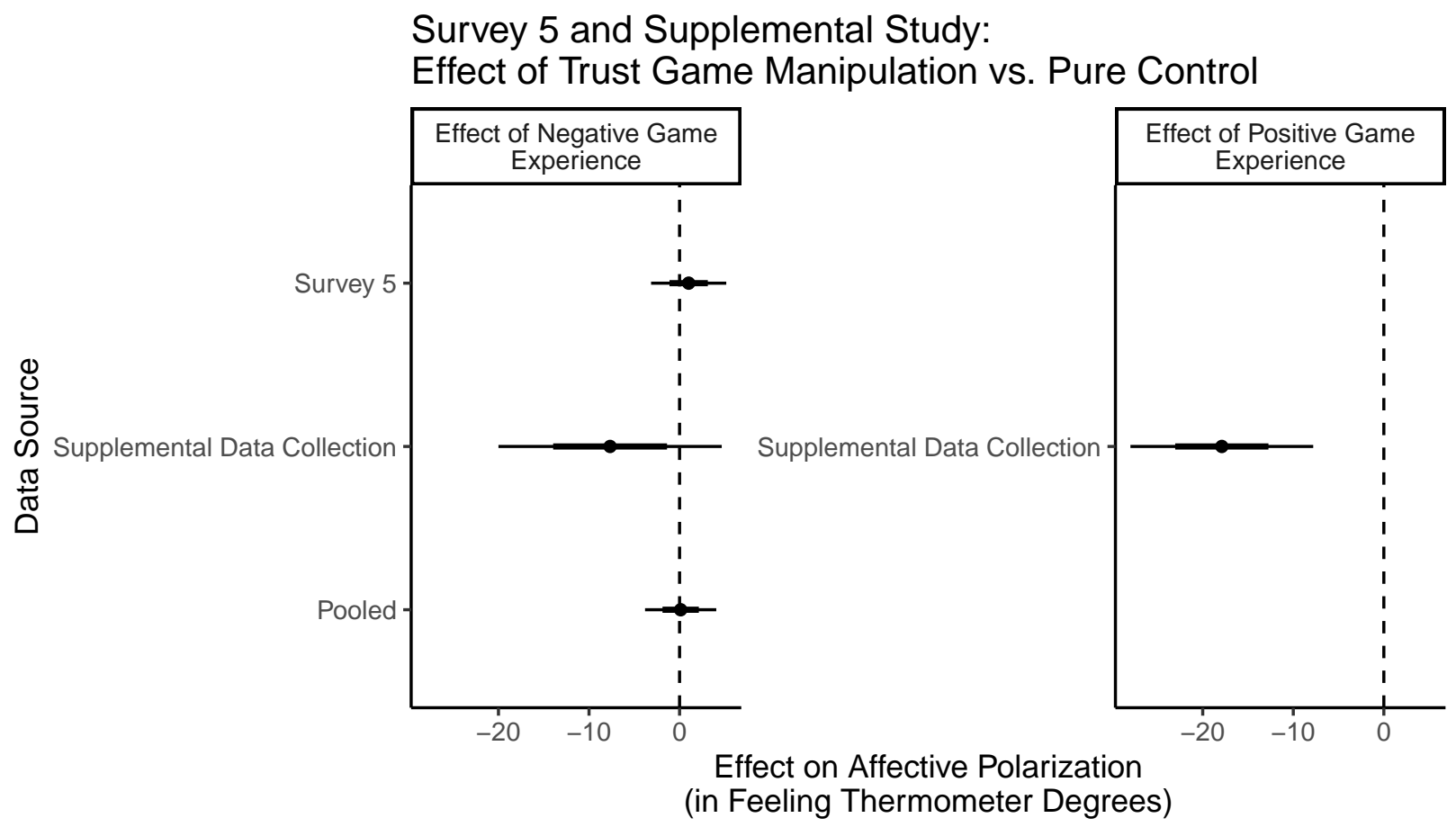

Notes: Point estimates are surrounded by standard errors (thick lines) and 95\% confidence intervals (thin lines). Point estimates are from multivariate regressions controlling for preregistered covariates (7-point partisanship scale and its absolute value (partisan strength)). Precision-weighting is used to pool across the two surveys.

The second panel of Figure A7 shows that, relative to a control group, the positive trust game condition dramatically decreases affective polarization, by 17.9 points $(S E=5.1, p<0.001)$. (There was no positive game experience condition in Survey 5.)

These results indicate that one should interpret the differences between the positive and negative trust game conditions presented in the main text as mainly reflecting the effects of a decrease in affective polarization brought on by the positive trust game.

This finding is consistent with the motivation for our research, which raises questions about 
whether decreasing affective polarization would meaningfully bolster norms or accountability. As compared to the interventions reported in Appendix $\mathrm{F}$, the positive trust game condition is perhaps the most powerful tool for reducing affective polarization available-yet we found it had no salutary downstream consequences for democratic norms or accountability.

There are several potential explanations for why the positive trust game condition so meaningfully decreases affective polarization while the negative trust game condition does not increase it. One possibility is that respondents already expect to be treated poorly by outpartisans, so the negative trust game provides no new or surprising information; the interaction proceeds exactly as they expect. However, respondents may not expect to be treated well by outpartisans, leading to the reductions in affective polarization observed in the positive trust game. Moreover, respondents in the positive game condition receive an actual financial bonus because of the putative outpartisan's behavior. Future research may wish to investigate these mechanisms.

\section{F Survey 5: Additional Details and Results}

Survey 5 was conducted in June 2021 using the online survey vendor Dynata, the same vendor as for surveys 1-4. In order to be eligible to participate, survey respondents needed to meet the same eligibility criteria as Surveys 1-4. In addition, survey respondents were ineligible if they already participated in Surveys 1-4 or were completing the survey on a mobile device. The study was pre-registered with the Center for Open Science. $N=2,504$ respondents completed the survey. The eligiblity rate was $30 \%$ for Survey 5 . This survey received IRB approval.

Table A3 presents the demographics of the survey samples relative to the 2019 Cooperative Congressional Election Study. In addition, to assess the political knowledge of the sample, we asked a political knowledge question that had previously been asked by the Pew Research Center ${ }^{15}$ We asked respondents "As you may know, presidents are chosen not by direct popular vote, but by the electoral college in which each state casts electoral votes. What determines the number of

\footnotetext{
${ }^{15}$ See https: / /perma.cc/7V2H-C856.
} 
electoral votes a state has?" The response options were "The number of seats the state has in the U.S. House and Senate" (correct), "The number of voters in the state," "The number of counties in the state," "Each state has the same number of electoral votes," and "Not sure." In the Pew sample, $39 \%$ answered correctly while in the Survey 5 sample, 37\% answered correctly. This suggests that the Survey 5 sample was, if anything, slightly less politically knowledgeable than the general population.

After collecting demographics, we randomly assigned respondents to one of four treatment conditions meant to induce variation in affective polarization or to a control group. This follows a similar design as Voelkel et al. (2021). The four treatment conditions were:

- Negative Trust Game. This is the same as in Surveys 1-4.

- Ahler and Sood (2018). Respondents are asked about their perceptions of the outparty's composition (e.g., What percentage of Republicans do you think are Evangelical?) and then have any misperceptions corrected.

- Lees and Cikara (2020). Respondents are asked about their perceptions of outparty opposition to a policy supported by the inparty and then have any misperceptions of the level of outparty support corrected. They are also shown that Democrats and Republicans both think similarly about this issue.

- Levendusky (2020). An outpartisan friendship is made salient through a writing prompt in which respondents are asked to reflect on a friend, relative, neighbor, or co-worker from the other party who they "like and respect a great deal."

\section{F.1 Results}

As shown in Figure 10, the Ahler and Sood (2018), Lees and Cikara (2020), and Levendusky (2020) treatments successfully reduced affective polarization compared to a control group ${ }^{16}$

\footnotetext{
${ }^{16}$ As we discuss further in Appendix $\mathrm{E}$, and consistent with the results reported there, the negative trust game had no effect on affective polarization (effect on mass public affective polarization of 0.03 standard deviations; $p=0.64$ ). We
} 
After asking the feeling thermometer questions necessary to measure affective polarization, we then asked two specific social distance items (respondents were randomly assigned to only one). Respondents were asked either to rate how likely they would be to hire a potential outpartisan job applicant (Iyengar and Westwood 2015) or to rate the attractiveness of an outpartisan dating profile (Nicholson et al.2016).

As shown in Figure 11 and Figure A8, we find no effect of exogenously reducing affective polarization on these specific social distance items. If anything, reducing affective polarization may have increased partisan employment based discrimination, with treatment effects primarily driven by the Lees and Cikara (2020) manipulation.

We then asked respondents the norms index, election override vignette, and violence justification index, mirroring Survey 4. As shown in Figure 11 and Figure A8, we find no effect of exogenously reducing affective polarization on these downstream political attitudes. The results from Survey 5 suggest that the null effects we observe in Surveys 1-4 on political attitudes is unlikely to be explained by the artificial nature of the trust game experience. Three alternative interventions produce similar null findings.

therefore do not include the negative trust game condition in Survey 5 in analyses examining effects on downstream outcomes. 


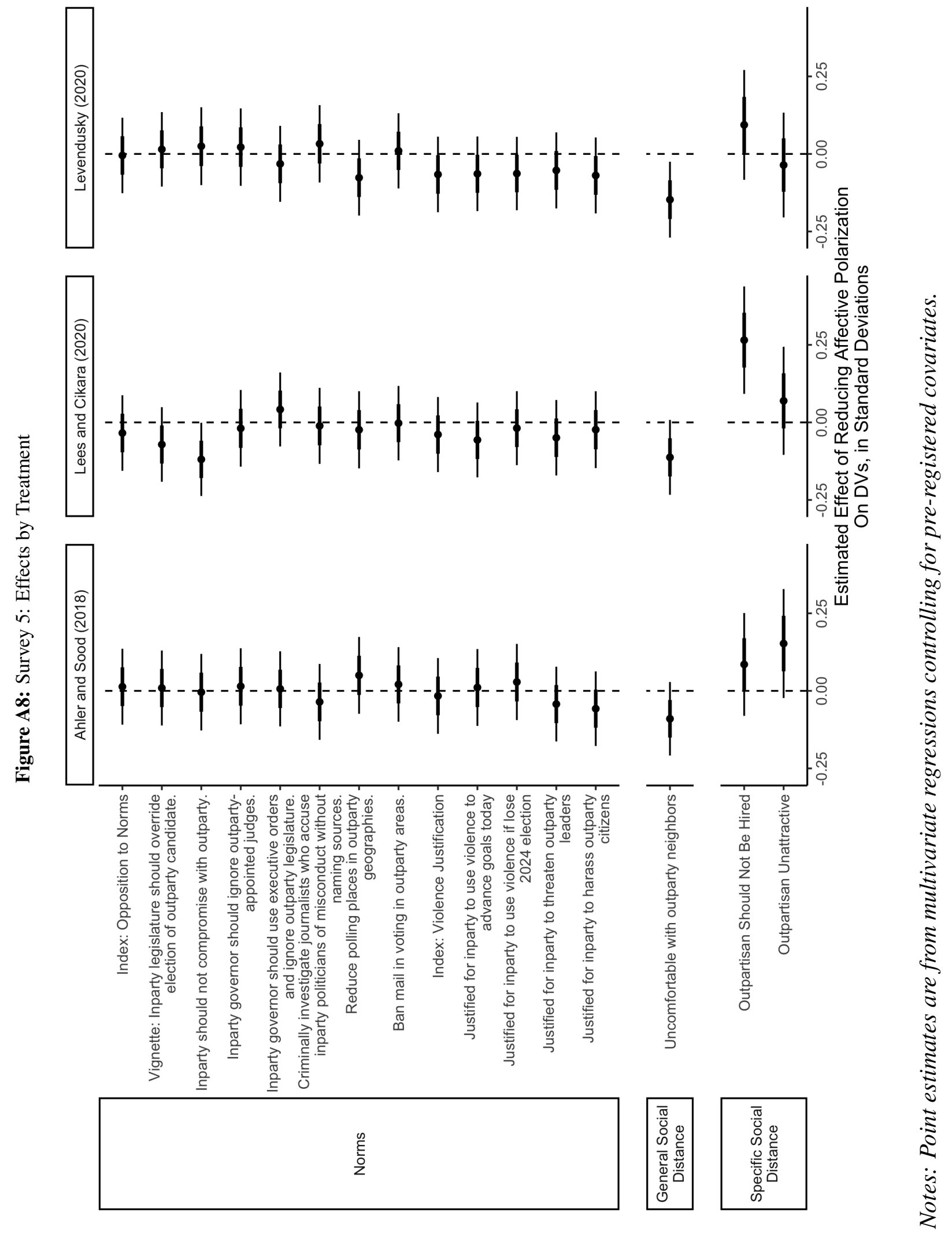




\section{F.2 Testing for Demand Effects}

Survey 5 also allows us to help assess whether experimenter demand effects may explain the null effects found in Surveys 1-4. A first possible concern regarding demand is that the trust game did not actually manipulate affective polarization at all, but that respondents thought researchers wanted to see this occur, and so answered the survey questions as if it had manipulated affective polarization. We see two reasons to be doubtful that demand contaminated the original results. First, the downstream effects on general social distance items suggest that affective polarization itself was successfully manipulated. Second, as discussed in the article and elaborated in Appendix E. the effects of the trust game are driven entirely by the positive treatment. If respondents simply "did what they thought researchers wanted," we would expect to see effects of both the negative and positive trust treatment. However, we only see effects of the positive treatment. With this said, Survey 5 helps address this further with its three new manipulations that reduce affective polarization. These three manipulations are less overt than the trust game manipulation and have also been demonstrated elsewhere to effectively reduce affective polarization. We continue to find consistent results with these three new manipulations.

To examine this further, we also added a series of distractor questions to Survey 5. Adding space in the form of non-political distractor questions between the manipulations and our dependent variables may reduce respondent suspicion. We asked respondents how favorably they felt towards 6 brands: Crest, Colgate, Honda, Toyota, Ford, and Apple. Moreover, we randomly assigned whether these distractor questions came between the affective polarization manipulation and the feeling thermometer manipulation checks or not. We find that treatment effects of the manipulations on reducing affective polarization are indistinguishable whether or not affective polarization is measured before or after the distractor questions $(p=0.98)$.

A second possible concern is that respondents' affective polarization was manipulated, but that demand concealed its effects. For this to have led to null results that concealed the literature's 
predicted effects, a positive trust game experience would need to have led respondents to choose to misrepresent their attitudes in a manner consistent with less accountability and being more antidemocratic, cancelling out the salutary effects of the reduced affective polarization. This seems unlikely, but we examined this by asking respondents what they thought the purpose of the study was. In particular, at the very end of Survey 5, we asked respondents "Thank you for participating in this study. Lastly, we want to know what you think the purpose of this study was. Please provide your best guess in the box below." We sampled 1,000 responses to this question and manually read through them. Only three comments linked the manipulations to downstream attitudes, all three of which focused on interpersonal attitudes, not the downstream political measures, and all three of which were focused on the trust game. The three comments were: (1) "To see if your feelings about Republicans change when individual Republicans treat you well." (2) "To see if the amount of money we were given or not given relates to our reports of how we perceive others. I do not believe there was really another player." (3) "my reaction to democratic partners that were rather selfish." Overall, while some respondents may have seen through the manipulation, the rate was quite low. Moreover, we received no such comments among respondents assigned to the three new interventions in Survey 5; and none of the three comments we received referenced impacts on downstream political attitudes. This suggests it is unlikely that respondents en masse decided to present their attitudes in a more anti-democratic manner as a result of the manipulations that decreased their affective polarization (the direction of bias that would be needed for the effect of affective polarization to be cancelled out by demand effects).

\section{G Statistically Summarizing the Results}

In the main text, we presented many estimates of the downstream effects of reducing affective polarization across many outcomes. Contrary to the expectation of a great deal of literature, nearly all of our findings were null. To summarize our results and illustrate the consistency of our null findings, here we compute the $t$-statistic associated with every result about the downstream effects 
of reducing affective polarization shown or discussed in the article.

Figure A9 shows histograms of all these $t$-statistics, grouped by area. Each histogram also has a dashed red line at -1.96 , the conventional statistical significance threshold (placed at -1.96 because all the coefficients are oriented to expect negative coefficients).

Figure A9: Histogram of $t$-statistics for results discussed and presented in this article, by outcome area

Histogram of t-statistics: Effects of Reducing Affective Polarization
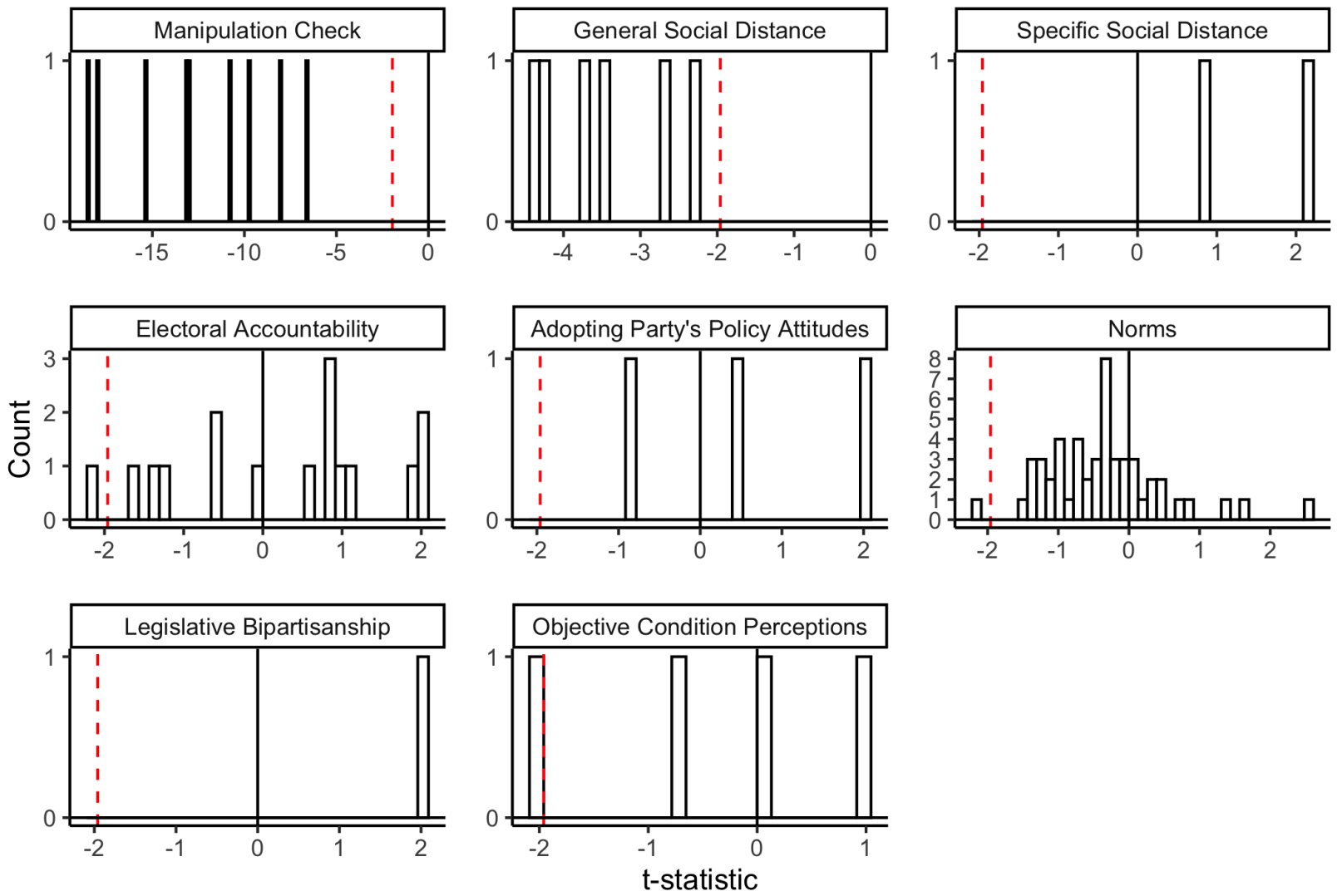

Notes: Histograms of t-statistics for results presented or discussed in this article, organized by outcome area. The red vertical line is at -1.96 , the conventional statistical significance threshold.

The first two panels show that the manipulation check items and the general social distance items all have highly statistically significant results. The third shows the null results in the specific social distance area. Finally, the distribution of $t$-statistics in the five political outcome areas are consistent with null results. Out of the 75 -statistics across these five areas, only 3 are large 
enough to just surpass statistical significance at the conventional level, in line with what would be expected by chance. Moreover each of these 3 statistically significant results are also accompanied by other clearly null results on either replications or closely related outcomes or tests, consistent with them being false positives ${ }^{17}$ Finally, when applying a False Discovery Rate adjustment to all the $p$-values across all the $t$-statistics, all the manipulation check and general social distance items remain significant at the conventional threshold, but none of the formerly significant outcomes in the other areas do.

These consistently null results cannot be explained by limited statistical power. Not only was our manipulation of affective polarization powerful (equal to approximately three decades of increased affective polarization) and our sample sizes large, we would expect to observe a larger proportion of statistically significant $t$-statistics were the null hypothesis wrong.

\section{H Comparing Experimental and Endogenous Estimates}

In this section, we show that the null results our experimental approach surfaced stand in contrast to large and positive-but misleading_results one would reach without it. In particular, for every result we discussed or presented in the article, we computed (1) the experimental estimate we show in the article (on standardized versions of each outcome) to (2) a non-causal endogenous estimate, computed by estimating the observational relationship between the difference in affective polarization equal to the size our experimental treatment created and each standardized outcome 18 This allows us to compare endogenous estimates of the form that the existing literature would compute to our causal estimates on the same scale.

\footnotetext{
${ }^{17}$ The first significant result is on Survey 1's "investigate journalists" norms item; however, this did not replicate in Survey 2 or 4 (Fig. A5). The second significant result is the decreased reward Members of Congress earned for casting congruent votes in Survey 3 (Fig. 5c), but the results for incongruent votes in Surveys 1 and Survey 3 (Fig. 5a and (5b) go in the opposite direction. The third significant result is the increased perception of the unemployment rate among Democrats, but we do not see these results for their perceptions of COVID deaths, nor for Republicans on either outcome (Fig. 9).

${ }^{18} \mathrm{We}$ compute the latter by running a regression of the outcome of interest on affective polarization, then multiplying the coefficient on affective polarization by the estimated effect of the intervention on affective polarization. This indicates what size of an effect from the experiment we would predict on the outcome were the endogenous relationship between affective polarization and the outcome causal.
} 
Figure A10 compares these endogenous estimates to our experimental estimates. The first panel, A10a, shows the general social distance outcomes. In this domain, where our argument predicts and we find causal effects of affective polarization, the endogenous correlational estimates and the experimental estimates are nearly identical.

Figure A10: Comparing Endogenous Estimates (x-axes) with Experimental Estimates (y-axes)

(a) Social Distance Outcomes

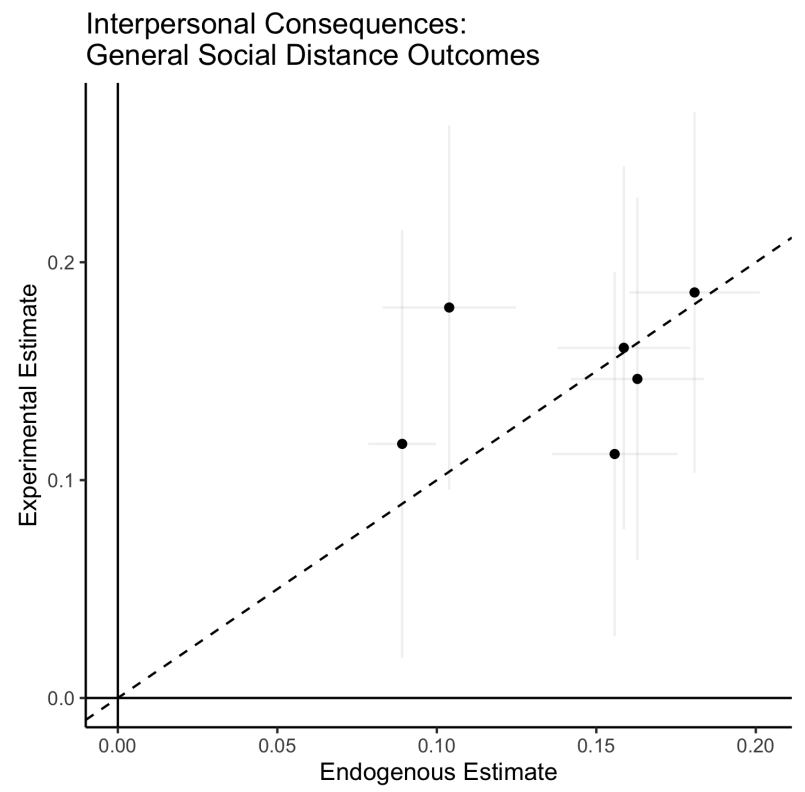

(b) Political Outcomes

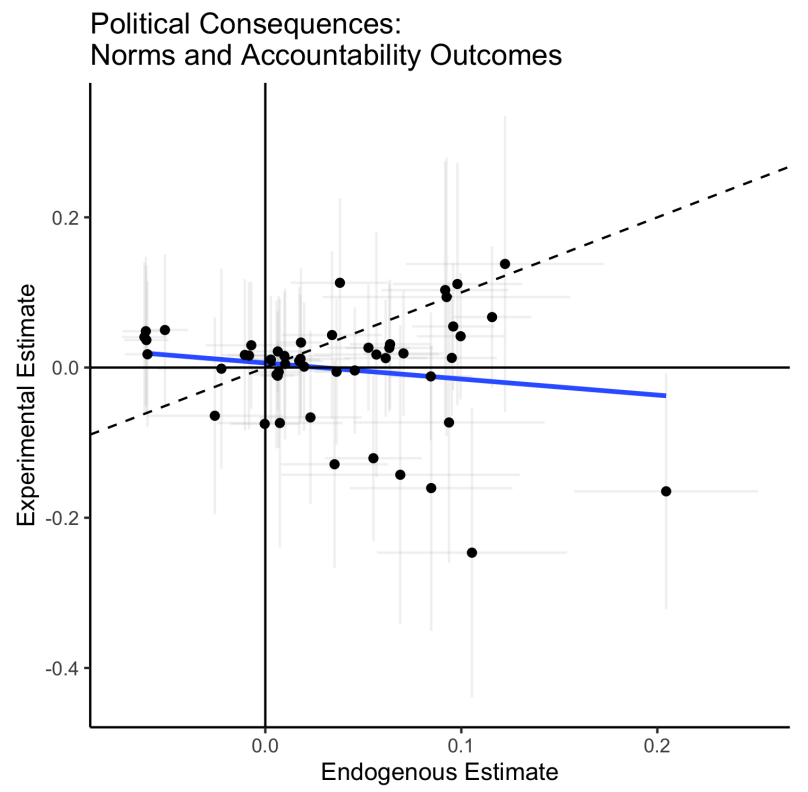

Notes: The $x$-axis value corresponds with the experimental estimate presented in the article on standardized versions of each outcome. The y-axis value is a non-causal endogenous estimate of the same effect, computed by estimating the observational relationship between an "increase" in affective polarization equal to the size of the effect of the trust game and each standardized outcome. 95\% confidence intervals in both regressions are shown surrounding the point estimates. The dotted line is a 45 degree line showing the slope $y=x$. Panel A10b shows a line of best fit in blue.

Figure $\mathrm{A} 10 \mathrm{~b}$ shows the same relationship for the political outcomes. Approximately $79 \%$ of the endogenous estimates are positive, meaning that a version of this article which followed existing literature in examining the observational relationship between affective polarization and these political outcomes would have reported nearly all positive results. However, only $46 \%$ of the experimental estimates are positive, essentially a coin flip. Moreover, $63 \%$ of the endogenous estimates are larger than the experimental estimates: the endogenous estimates consistently overestimate the causal effects of affective polarization. Finally, the blue line of best fit shows that 
there is no relationship between the size of the experimental and the endogenous estimates.

These results provide evidence for the alternative relationship between affective polarization and downstream political choices we hypothesized in Figure 1b. Affective polarization correlates with a number of other constructs, including the intensity of partisanship, policy preferences, etc., perhaps as it may summarize these considerations. However, because these other constructs are also likely to correlate with downstream political choices, they represent omitted variables that inflate the correlation between affective polarization and these downstream choices. These results provide caution for analysts interested in affective polarization's impacts, as it makes it difficult, if not impossible, for analysts to isolate the downstream causal effects of affective polarization from the effects of these other considerations in observational data, and very likely to overestimate them.

\section{References for Online Appendix}

Ahler, Douglas J. and Gaurav Sood. 2018. "The parties in our heads: Misperceptions about party composition and their consequences." Journal of Politics 80(3):964-981.

Iyengar, Shanto and Sean J. Westwood. 2015. "Fear and Loathing across Party Lines: New Evidence on Group Polarization.” American Journal of Political Science 59(3):690-707.

Lee, David S. 2009. "Training, wages, and sample selection: Estimating sharp bounds on treatment effects." The Review of Economic Studies 76(3):1071-1102.

Lees, Jeffrey and Mina Cikara. 2020. "Inaccurate group meta-perceptions drive negative out-group attributions in competitive contexts.” Nature Human Behaviour 4(3):279-286.

Levendusky, Matthew S. 2020. "Our common bonds: Using what Americans share to help bridge the partisan divide." Unpublished Manuscript .

Nicholson, Stephen P, Chelsea M Coe, Jason Emory and Anna V Song. 2016. "The politics of beauty: The effects of partisan bias on physical attractiveness.” Political Behavior 38(4):883898. 